HD-TVP-97/03

\title{
A Numerical Study of an Expanding Plasma of Quarks in a Chiral Model
}

\author{
P. Rehberg and J. Hüfner \\ Institut für Theoretische Physik, Universität Heidelberg, \\ Philosophenweg 19, D-69120 Heidelberg, Germany
}

\begin{abstract}
We numerically solve the transport equations for a quark gas described by the the Nambu-Jona-Lasinio model. The mean field equations of motion, which consist of the Vlasov equation for the density and the gap equation for the mean field, are discussed, and energy and momentum conservation are proven. Numerical solutions of the partial differential equations are obtained by applying finite difference methods. For an expanding fireball of the light quark mass evolves from small values initially to the value of $350 \mathrm{MeV}$. This leads to a depletion of the high energy part of the quark spectrum and an enhancement at low momenta. When collisions are included one obtains an equation of the Boltzmann type, where the transition amplitudes depend on the properties of the medium. These equations are given for flavor $S U(3)$, i.e. including strangeness. They are solved numerically in the relaxation time approximation and the time evolution of various observables is given. Medium effects in the relaxtion times do not significantly influence the shape of the spectra. The mass of the strange quark changes little during the expansion. The strangeness yield and the slope temperatures of the final spectra are studied as a function of the size of the initial fireball.
\end{abstract}

PACS numbers: 25.75.-q, 12.38.Mh, 12.39.Fe, 52.25.Dg

Typeset using REVTEX 


\section{INTRODUCTION}

Deconfinement and chiral symmetry determine the properties of strongly interacting matter at high temperatures and densities to a large extent. While deconfinement is yet far from being understood, chiral symmetry restoration and the resulting effects can be shown to play the dominant role for the low energy sector of strong interactions and can be well studied within phenomenological models. However, most of these studies are restricted to the case of thermal equilibrium. Since heavy ion experiments are non-equilibrium phenomena, there is a need for incorporating chiral symmetry breaking and restoration into a non-equilibrium theory. This is the purpose of this paper.

In order to develop a theoretical model for the evolution of heavy ion collisions including a dynamical breaking of chiral symmetry, one has to choose a model Lagrangian for the underlying interaction. Here we employ the Nambu-Jona-Lasinio (NJL) model in its two and three flavor versions [1]. Although nonconfining and nonrenormalizable, this model describes well the properties of light hadrons in the vacuum. Recent lattice results indicate that also at high temperatures around the phase transition the temperature dependence of the condensate and of certain masses is correctly reproduced by the model [2]. Its relative simplicity is an additional good reason to start from this effective interaction. The NJL Lagrangian is used in the

Schwinger-Keldysh formalism for non-equilibrium Green functions and equations of motion for the particle densities are obtained. Since their derivation is difficult, we give only a brief outline of this calculation and refer for more details about this subject to Refs. [3, 4].

In the present work we focus on the numerical solution of the transport equations. To this end we firstly investigate the mean field equations of motion, i. e. the Vlasov equation, which describes the evolution of the density evolving in a mean field, and the gap equation, which describes the selfconsistent coupling of the mean field to the density. We show that particle number, energy and momentum are conserved. The latter two conservation laws rely on the existence of a potential energy, which we derive. The Vlasov equation is a partial differential equation, which we numerically solve using a finite difference method. For simplicity, we confine ourselves to spherically symmetric systems. The numerical solution of the Vlasov equation is 
presented and the physical effects generated by the mean field are discussed.

In a second part, binary collisions are included into the equations of motion. Since we also want to study the generation of of strange quarks via the processes $u \bar{u} \rightarrow s \bar{s}$ and $d \bar{d} \rightarrow s \bar{s}$, we use the three flavor version of the NJL model. After sketching the derivation of an equation of the Boltzmann type including medium effects, we solve this equation using a relaxtion time approach. The relaxation times entering this ansatz are computed from cross sections, which depend on temperature and are given in Ref. [5]. As for the Vlasov equation, a numerical solution of the Boltzmann equation is given.

We investigate the how the numerical results change when the medium effects are switched off in the relaxation times and in the mean field. We investigate also how a variation of the size of the initial system influences the final particle spectra and multiplicities. In particular, we study the chemical equilibration of strange quarks as the initial fireball grows larger.

To our knowledge, it is the first time that a kinetic equation for a strongly interacting system is solved where the input - mean fields and collision terms are derived consistently from an underlying relativistic Lagrangian and where all necessary medium effects are included. In particular the underlying symmetry here chiral symmetry - is consistently preserved. Also in the numerical solution we have chosen an unconventional way: Solving a partial differential equation instead of the test particle method. Of course, also a price has to be paid: The investigated system, a quark gas which, at the present stage, does not have the possibility of hadronization, is rather unrealistic and any comparison of our results with data from heavy ion collisions contains large uncertainties. Therefore the results of the paper are important for further theoretical work.

\section{THE VLASOV EQUATION FOR THE TWO FLAVOR NJL MODEL}

In this section, we give a brief derivation of the Vlasov equation and a short introduction to the NJL model in its two flavor version and discuss the conservation laws. Then we outline the numerical methods used for solving the Vlasov equation and present results for one initial condition. 


\section{A. Derivation of the Vlasov Equation}

The starting point of our discussion is the equation of motion for the real time Green function $G^{<}$, which in coordinate space is defined via [6]

$$
G^{<}(x, y)=i\langle\bar{\psi}(y) \psi(x)\rangle
$$

where $\psi(x)$ is is the destruction operator for a quark at space-time point $x, \bar{\psi}(y)=$ $\psi^{\dagger}(y) \gamma^{0}$ and $\langle\ldots\rangle$ denotes the average over the ensemble under consideration. Note that $G^{<}$is a matrix in Dirac space as well as in color and flavor space. At the mean field level, the equation of motion for $G^{<}(x, y)$ can be written as [6, []

$$
\left(i \not \partial_{x}-m_{q}(x)\right) G^{<}(x, y)=0
$$

In this equation, $m_{q}(x)$ is a space-time dependent mass, which is given as the sum of the bare mass and the Hartree part of the self energy [4,6]. We transform Eq. (2.1) to phase space with the help of the Wigner transformation

$$
G^{<}(x, p)=\int d^{4} u e^{i p u} G^{<}\left(x+\frac{u}{2}, x-\frac{u}{2}\right)
$$

and introduce the quasiparticle approximation for $G^{<}(x, p)$ [1, 6]:

$$
\begin{array}{r}
G^{<}(x, p)=\frac{i \pi}{2 E(x, \vec{p})} \frac{\delta_{f f^{\prime}} \delta_{c c^{\prime}}}{N_{c} N_{f}}\left(\not p+m_{q}(x)\right)\left[\delta\left(p_{0}-E(x, \vec{p})\right) n_{q}(x, \vec{p})\right. \\
\left.-\delta\left(p_{0}+E(x, \vec{p})\right)\left(2 N_{c} N_{f}-n_{\bar{q}}(x,-\vec{p})\right)\right],
\end{array}
$$

where $E(x, \vec{p})=\sqrt{\vec{p}^{2}+m_{q}^{2}(x)}$. In Eq. (2.4) color and flavor indices are shown explicitly and it is assumed that the particle distributions for each individual degree of freedom are independent of color, flavor and spin, so that they can be expressed by $n_{q}(x, \vec{p})$ and $n_{\bar{q}}(x, \vec{p})$, which are the total number of quarks and antiquarks per phase space cell, respectively. Inserting Eq. (2.4) into Eq. (2.1) and keeping only the leading order in a gradient expansion leads to the Vlasov equation

$$
\left[\partial_{t}+\vec{v}(x, \vec{p}) \vec{\partial}_{x}-\vec{\partial}_{x} E(x, \vec{p}) \vec{\partial}_{p}\right] n_{q}(x, \vec{p})=0
$$

which is valid for both $n_{q}$ and $n_{\bar{q}}$. Here the velocity is defined by

$$
\vec{v}(x, \vec{p})=\frac{\vec{p}}{E(x, \vec{p})}=\vec{\partial}_{p} E(x, \vec{p}) .
$$

Note that Eq. (2.5) has been derived without making assumptions about the interaction. It is thus generic for any model [6]. 


\section{B. The Mass of the Constituent Quark in the NJL Model}

In order to be complete, Eq. (2.5) has to be supplemented by an equation for the quark mass $m_{q}(x)$, which in turn has to be given by some model for the interaction. In the following we will use the $S U_{f}(2)$ version of the NJL model, which is defined by the Lagrangian [1]

$$
\mathcal{L}=\bar{\psi}\left(i \not \partial-m_{0 q}\right) \psi+G\left[(\bar{\psi} \psi)^{2}+\left(\bar{\psi} i \gamma_{5} \vec{\tau} \psi\right)^{2}\right]
$$

where $m_{0 q}$ is the current quark mass, $G$ a coupling constant and $\vec{\tau}$ the Pauli matrices in flavor space. For the calculation of the space-time dependent mass $m_{q}(x)$, we confine ourselves to a selfconsistent Hartree approximation, which is the lowest order in an $1 / N_{c}$ expansion [8]. In this approximation, $m_{q}(x)$ is given by [0, 4$]$

$$
m_{q}(x)=m_{0 q}-2 i G \operatorname{Tr} i G^{<}(x, x),
$$

where the trace runs over spin, color and flavor degrees of freedom. After inserting Eq. (2.4) into Eq. (2.8) and performing the $p_{0}$ integration, one obtains the NJL gap equation [1]:

$$
m_{q}(x)=m_{0 q}+2 G m_{q}(x) \int_{|\vec{p}|<\Lambda} \frac{d^{3} p}{(2 \pi)^{3}} \frac{2 N_{c} N_{f}-n_{q}(x, \vec{p})-n_{\bar{q}}(x, \vec{p})}{E(x, \vec{p})} .
$$

Since the NJL model is not renormalizable, an $O(3)$ cutoff $\Lambda$ has been introduced which makes the integral finite.

\section{Conservation Laws}

The coupled equations (2.5) and (2.9) describe the time evolution of the quark plasma in phase space. Before we describe the numerical solution of these equations, we prove that they conserve the total number of quarks and antiquarks

$$
\mathcal{N}_{q}=\int \frac{d^{3} x d^{3} p}{(2 \pi)^{3}} n_{q}(x, \vec{p}) \quad \mathcal{N}_{\bar{q}}=\int \frac{d^{3} x d^{3} p}{(2 \pi)^{3}} n_{\bar{q}}(x, \vec{p})
$$

the total momentum

$$
\overrightarrow{\mathcal{P}}=\int \frac{d^{3} x d^{3} p}{(2 \pi)^{3}} \vec{p}\left(n_{q}(x, \vec{p})+n_{\bar{q}}(x, \vec{p})\right),
$$


and the total energy $\mathcal{E}_{\text {tot }}$, which will be defined later.

The time derivative of the total quark number is given, after inserting (2.5) into $(2.10)$, by

$$
\frac{d \mathcal{N}_{q}}{d t}=\int \frac{d^{3} x d^{3} p}{(2 \pi)^{3}}\left(-\vec{v}(x, \vec{p}) \vec{\partial}_{x}+\vec{\partial}_{x} E(x, \vec{p}) \vec{\partial}_{p}\right) n_{q}(x, \vec{p})
$$

After integrating the second term by parts, the two volume integrals in Eq. (2.12) cancel. The remaining surface terms also vanish, if $n_{q}(x, \vec{p})$ vanishes both for high momenta and large values of $|\vec{x}|$. Thus the number of quarks and analogously the number of antiquarks is conserved. Note that the conservation of the particle numbers can be proven independently of the functional form of the interaction.

The proof of energy conservation is less trivial and depends crucially on how $m_{q}(x)$ is calculated from a dynamical equation. This is obvious since there are forms of interactions, e.g. explicitly time dependent external fields, which do not conserve energy. In order to prove energy conservation for the Vlasov equation in the NJL model, we define the kinetic energy as

$$
\mathcal{E}_{\text {kin }}=\int \frac{d^{3} x d^{3} p}{(2 \pi)^{3}} E(x, \vec{p})\left(n_{q}(x, \vec{p})+n_{\bar{q}}(x, \vec{p})\right) .
$$

After inserting the Vlasov equation (2.5), one obtains

$$
\begin{aligned}
\frac{d \mathcal{E}_{\text {kin }}}{d t}=\int \frac{d^{3} x d^{3} p}{(2 \pi)^{3}}[ & \partial_{t} E(x, \vec{p})\left(n_{q}(x, \vec{p})+n_{\bar{q}}(x, \vec{p})\right) \\
& \left.+\left(-\vec{p} \vec{\partial}_{x}+m_{q}(x) \vec{\partial}_{x} m_{q}(x) \vec{\partial}_{p}\right)\left(n_{q}(x, \vec{p})+n_{\bar{q}}(x, \vec{p})\right)\right] .
\end{aligned}
$$

The second term on the right hand side can be transformed to surface integrals and does not contribute. The first term can be rewritten to give

$$
\frac{d \mathcal{E}_{\text {kin }}}{d t}=\int d^{3} x m_{q}(x) \partial_{t} m_{q}(x) \int \frac{d^{3} p}{(2 \pi)^{3}} \frac{n_{q}(x, \vec{p})+n_{\bar{q}}(x, \vec{p})}{E(x, \vec{p})} .
$$

In order to proceed further, one has to make the crucial assumption that the system does not contain quarks with momentum larger than $\Lambda$. Then the momentum integral in Eq. (2.15) can be transformed by using the gap equation (2.9):

$$
\int \frac{d^{3} p}{(2 \pi)^{3}} \frac{n_{q}(x, \vec{p})+n_{\bar{q}}(x, \vec{p})}{E(x, \vec{p})}=2 N_{c} N_{f} \int_{|\vec{p}|<\Lambda} \frac{d^{3} p}{(2 \pi)^{3}} \frac{1}{E(x, \vec{p})}+\frac{m_{0 q}-m_{q}}{2 G m_{q}} .
$$

The right hand side now depends on $x$ only through the mass, so that we can define an effective potential $\mathcal{V}_{\text {eff }}$ via 


$$
\int \frac{d^{3} p}{(2 \pi)^{3}} \frac{n_{q}(x, \vec{p})+n_{\bar{q}}(x, \vec{p})}{E(x, \vec{p})}=-\frac{1}{m_{q}(x)} \frac{d \mathcal{V}_{\mathrm{eff}}\left(m_{q}(x)\right)}{d m_{q}}
$$

The effective potential is given explicitly by [9,10,

$$
\begin{aligned}
\mathcal{V}_{\mathrm{eff}}\left(m_{q}\right) & =\int^{m_{q}} d \mu\left(\frac{\mu-m_{0 q}}{2 G}-\frac{2 N_{c} N_{f} \mu}{\pi^{2}} \int_{0}^{\Lambda} d p \frac{p^{2}}{\sqrt{p^{2}+\mu^{2}}}\right) \\
& =\frac{\left(m_{q}-m_{0 q}\right)^{2}}{4 G}+\frac{N_{c} N_{f} m_{q}^{4}}{8 \pi^{2}} \operatorname{arsinh}\left(\frac{\Lambda}{m_{q}}\right)-\frac{N_{c} N_{f} \Lambda}{8 \pi^{2}} \sqrt{\Lambda^{2}+m_{q}^{2}}\left(2 \Lambda^{2}+m_{q}^{2}\right) .
\end{aligned}
$$

Note that this result has been already derived in Ref. [9] from a field theoretical point of view. After inserting Eq. (2.17) into Eq. (2.15), one obtains

$$
\frac{d \mathcal{E}_{\mathrm{kin}}}{d t}=-\frac{d}{d t} \int d^{3} x \mathcal{V}_{\mathrm{eff}}\left(m_{q}(x)\right)
$$

By defining the potential energy $\mathcal{E}_{\text {pot }}$ as

$$
\mathcal{E}_{\text {pot }}=\int d^{3} x \mathcal{V}_{\text {eff }}\left(m_{q}(x)\right)
$$

and the total energy as $\mathcal{E}_{\text {tot }}=\mathcal{E}_{\text {kin }}+\mathcal{E}_{\text {pot }}$, Eq. (2.19) gives

$$
\frac{d \mathcal{E}_{\text {tot }}}{d t}=0
$$

i. e. the total energy is conserved. It can also be read off from Eq. (2.19), that the quantity

$$
\epsilon(x)=\left[\int \frac{d^{3} p}{(2 \pi)^{3}} E(x, \vec{p})\left(n_{q}(x, \vec{p})+n_{\bar{q}}(x, \vec{p})\right)\right]+\mathcal{V}_{\mathrm{eff}}\left(m_{q}(x)\right)
$$

has the meaning of an energy density. We note again, that Eq. (2.21) can only be obtained by assuming that no particles with momentum larger than $\Lambda$ appear in the system. This somewhat artificial restriction arises as a consequence of the introduction of the $O(3)$ cutoff into the NJL model. The form of $\mathcal{V}_{\text {eff }}$ as a function of the constituent quark mass $m_{q}$, as evaluated from Eq. (2.18), is shown in Fig. 1. $\mathcal{V}_{\text {eff }}$ has a minimum at the constituent quark mass in the vacuum, since, according to Eq. (2.17), $\mathcal{V}_{\text {eff }}$ arises as an integral over the vacuum gap equation. In the vacuum, the gap equation is thus equivalent to the condition that the energy density, as defined by Eq. (2.22), is minimized. In Fig. 1, we have also added a constant to Eq. (2.18), so that $\mathcal{V}_{\text {eff }}=0$ at the vacuum quark mass and thus vanishes in the vacuum. 
As for the case of energy conservation, momentum conservation can also be proven only if the interaction is specified. The time derivative of the total momentum is obtained by inserting (2.5) into (2.11). After performing an integration by parts and dropping the surface terms, one obtains

$$
\frac{d \overrightarrow{\mathcal{P}}}{d t}=-\int \frac{d^{3} x d^{3} p}{(2 \pi)^{3}}\left(n_{q}(x, \vec{p})+n_{\bar{q}}(x, \vec{p})\right) \vec{\partial}_{x} E(x, \vec{p})
$$

The structure of the momentum integral is now the same as in Eq. (2.15). One thus has

$$
\frac{d \overrightarrow{\mathcal{P}}}{d t}=\int d^{3} x \vec{\partial}_{x} \mathcal{V}_{\mathrm{eff}}\left(m_{q}(x)\right)=\overrightarrow{0}
$$

i. e. the total momentum is conserved. As Eq. (2.21), Eq. (2.24) is only valid strictly if no particles with momentum larger than $\Lambda$ are present.

\section{Numerical Methods}

The Vlasov equation (2.5) is a first order partial differential equation, which in general depends on the seven variables $t, \vec{x}$ and $\vec{p}$. The usual solution method for this equation is the test particle method [11,12], which is based on a solution of the characteristic equations. Here we do not follow this way, but rather solve the equation by a finite difference method in order to study the advantages and disadvantages of this approach. In order to reduce the numerical effort, we assume that the system is spherically symmetric. This, in turn, implies that $n_{q}(x, \vec{p})$ can only depend on time and the three variables

$$
r=|\vec{x}|, \quad \quad \quad \quad \quad=|\vec{p}|, \quad \frac{\vec{x} \vec{p}}{|\vec{x}||\vec{p}|} .
$$

The last of these variables, $\eta$, is the cosine of the angle enclosed between $\vec{x}$ and $\vec{p}$. With the variables of Eq. (2.25), Eq. (2.5) transforms to

$$
\partial_{t} n_{q}=\frac{1}{E}\left[\eta\left(m_{q} \partial_{r} m_{q} \partial_{p} n_{q}-p \partial_{r} n_{q}\right)+\left(1-\eta^{2}\right)\left(\frac{m_{q} \partial_{r} m_{q}}{p}-\frac{p}{r}\right) \partial_{\eta} n_{q}\right],
$$

where the arguments of $n_{q}, m_{q}$ and $E$ have been dropped for simplicity. In order to solve Eq. (2.26), we use a finite difference scheme. In detail this proceeds as 
follows: Firstly, the variables $r, p, \eta$ are discretized equidistantly according to the prescription

$$
\begin{aligned}
r_{i} & =i r_{\max } / N_{r}, & i & =1 \ldots N_{r} \\
p_{j} & =j p_{\max } / N_{p}, & j & =1 \ldots N_{p} \\
\eta_{k} & =\left[2(k-1) /\left(N_{\eta}-1\right)\right]-1, & k & =1 \ldots N_{\eta} .
\end{aligned}
$$

This parametrization avoids the coordinate singularities of Eq. (2.26) occurring at $r=0$ and $p=0$. The time derivative of $n_{q}$ at time $t_{n}$ is replaced by

$$
\partial_{t} n_{q}(t, r, p, \eta) \rightarrow \frac{1}{\Delta t}\left[n_{q}\left(t_{n+1}, r_{i}, p_{j}, \eta_{k}\right)-n_{q}\left(t_{n}, r_{i}, p_{j}, \eta_{k}\right)\right] .
$$

In order to achieve numerical stability, we use a fully implicit procedure for the representation of $\partial_{r} n_{q}$ at time $t_{n}$,

$$
\partial_{r} n_{q}(t, r, p, \eta) \rightarrow\left\{\begin{array}{cl}
\frac{1}{\Delta r}\left[n_{q}\left(t_{n+1}, r_{i+1}, p_{j}, \eta_{k}\right)-n_{q}\left(t_{n+1}, r_{i}, p_{j}, \eta_{k}\right)\right], & i=1 \\
\frac{1}{2 \Delta r}\left[n_{q}\left(t_{n+1}, r_{i+1}, p_{j}, \eta_{k}\right)-n_{q}\left(t_{n+1}, r_{i-1}, p_{j}, \eta_{k}\right)\right], & 1<i<N_{r} \\
\frac{1}{\Delta r}\left[n_{q}\left(t_{n+1}, r_{i}, p_{j}, \eta_{k}\right)-n_{q}\left(t_{n+1}, r_{i-1}, p_{j}, \eta_{k}\right)\right], & i=N_{r}
\end{array}\right.
$$

and analogous prescriptions for $\partial_{p} n_{q}$ and $\partial_{\eta} n_{q}$. Note that this implies open boundary conditions, i.e. we assume that $n_{q}$ can be continued smoothly to points outside the grid. The time update for $n_{q}$ is done using an operator splitting scheme [13], i. e. we sequentially perform the three update steps which would arise if only the terms proportional to $\partial_{r} n_{q}, \partial_{p} n_{q}$ and $\partial_{\eta} n_{q}$ were present on the right hand side of Eq. (2.26). Technically, each update consists of the solution of tridiagonal linear systems, which can be implemented efficiently using the Thomas algorithm [13]. With these ingredients at hand, the calculation proceeds as follows:

i. Initialize $n_{q}$ and $n_{\bar{q}}$ according to some given initial conditions.

ii. Compute $m_{q}$ at each space point.

iii. Compute physically relevant quantities like particle density, energy density etc. and store them for later evaluation.

iv. Calculate $n_{q}$ and $n_{\bar{q}}$ on the next time slice. 


\section{v. Proceed with step iil.}

The calculation stops at that time, when the system has become a system of noninteracting particles. A sufficient criterion for this is that the quark mass comes close to its vacuum value everywhere.

\section{E. Numerical Results}

In this section we discuss our numerical results for the $S U_{f}(2)$ Vlasov equation. The model parameters chosen are $m_{0 q}=5.0 \mathrm{MeV}, \Lambda=653 \mathrm{MeV}$ and $G \Lambda^{2}=2.10$ 14. The initial conditions correspond to a spherically symmetric fireball around the origin with baryon number zero:

$$
n_{q}(x, \vec{p})=n_{\bar{q}}(x, \vec{p})=\exp \left(-\frac{r^{2}}{2 r_{0}^{2}}\right) \frac{2 N_{c} N_{f}}{\exp \left(\sqrt{p^{2}+m_{i}^{2}} / T_{0}\right)+1} f_{c}\left(\frac{p+p_{c}-\Lambda}{\delta p}\right)
$$

with a cutoff function $f_{c}(x)=(1-\tanh x) / 2$. The first factor of Eq. (2.30) describes the spatial shape of the system. The second factor is a Fermi distribution, which describes the distribution in momentum space. The mass $m_{i}$ is chosen to be the thermal mass corresponding to $T_{0}$, i. e. we have complete thermal equilibrium in the centre of the fireball. The third factor serves to cut off high momenta. This is necessary, since the boundary condition of Eq. (2.29) assumes that the particle distribution can be continued smoothly beyond the grid boundary. In order to avoid the cutoff artifacts which were discussed in Section $\Pi$, and to obtain exact energy conservation, it is thus necessary to cut off the distribution smoothly. The function $f_{c}$ is designed to interpolate smoothly between the values $f_{c}\left[\left(p+p_{c}-\Lambda\right) / \delta p\right] \approx 1$ for $p \ll \Lambda-p_{c}$ and $f_{c}\left[\left(p+p_{c}-\Lambda\right) / \delta p\right]=0$ for $p \gg \Lambda-p_{c}$, the drop between these two values taking place within an interval $\delta p$ around $p=\Lambda-p_{c}$. The standard parameters chosen for Eq. (2.30) are $r_{0}=3 \mathrm{fm}$ for the initial radius and $T_{0}=240 \mathrm{MeV}$ for the initial temperature. The parameters entering the momentum cutoff factor are $p_{c}=100 \mathrm{MeV}, \delta p=20 \mathrm{MeV}$.

The maximal values of $r$ and $p$ on the grid are $r_{\max }=10 \mathrm{fm}, p_{\max }=\Lambda$. The size of the grid is $N_{r}=N_{p}=100, N_{\eta}=50$. 
The fireball described at $t=0$ by Eq. (2.30) expands for $t>0$ due to thermal motion. After a certain time the density of particles is sufficiently low that the system behaves as a system of non interacting particles. This is demonstrated in Fig. 2, where the quark density at various times is shown as a function of $r$. At $t=0$, the density is described by the factor $\exp \left(-r^{2} / 2 r_{0}^{2}\right)$ in Eq. (2.30). At later times, the system expands and the density drops. Although Fig. 2 2 shows the density up to $t=8 \mathrm{fm}$ only, the calculation has been extended to $t=17 \mathrm{fm} / c$, when the density practically vanishes.

The quark mass $m_{q}(r)$, shown in Fig. 3, behaves in a similar way. At $t=0$ the quark mass forms a "potential well" due to the high density in the centre. As the density drops, the quark mass approaches its vacuum value of $m_{q \text {,vac }}=312 \mathrm{MeV}$. For $t \geq 17 \mathrm{fm} / c, m_{q}(r)$ is practically constant. This leads to a vanishing mean field term in Eq. (2.5) and the system decouples.

The final "observed" momentum spectra are calculated using the observation that there exist closed surfaces in coordinate space with the properties that (i) at $t=0$ all particles are contained within this surface, (ii) at time $t=\infty$ all particles are outside the surface and (iii) outside the surface the system behaves effectively as a system of non-interacting particles. The particle spectra then can be obtained by integrating the flux through such a surface. Choosing the surface to be a sphere with radius $r_{1}$, one obtains

$$
\frac{d \mathcal{N}_{q}}{d^{3} p}=\frac{r_{1}^{2}}{(2 \pi)^{3}} \int_{0}^{\infty} d t \int_{r=r_{1}} d \Omega n_{q} v_{\perp}
$$

where $v_{\perp}$ is the velocity component perpendicular to the surface. Since in practice the time integration in Eq. (2.31) can only be extended to finite times, we add also the (small) contribution of those particles, which still remain inside the surface $r=r_{1}$ when the calculation stops. In Fig. 4, the momentum spectra of quarks are shown for the initial and final states. At $t=0$, the spectra are governed by the Fermi part of Eq. (2.30) up to $p \approx 550 \mathrm{MeV}$, where the distribution is cut off by the factor $f_{c}\left[\left(p+p_{c}-\Lambda\right) / \delta p\right]$ in Eq. (2.30). During the evolution, the number of particles with low momenta is enhanced, while the high momentum region is depleted, since the generation of a dynamical mass during the evolution slows particles down and thus enhances the low momentum region of the spectrum.

The time development of the total energy is interesting in two respects: firstly 
as an accuracy check to the program and secondly for a study of the importance of the potential energy, which is usually neglected. The time behaviour of $\mathcal{E}_{\text {kin }}$ and $\mathcal{E}_{\text {tot }}$ is given in Fig. 5. As was detailled in Section $\Pi$ I , the potential energy is positive and vanishes in the vacuum. This behaviour can be observed in Fig. 5: At small times, when the system consists of low mass quarks, the potential energy is maximal and contributes about 5\% to the total energy. During the expansion, the quark mass approaches its vacuum value and the the potential energy goes to zero. Concomitantly, the kinetic energy rises. Calculating kinetic and potential energies numerically, we find that energy is conserved to an accuracy of $1 \%$. We also obtain particle number conservation with an accuracy of $6 \%$. Both results point to a numerical accuracy of the order of $5-10 \%$. The total momentum, on the other hand, vanishes identically due to the spherical geometry.

\section{THE BOLTZMANN EQUATION FOR THE THREE FLAVOR NJL MODEL}

The results of the previous section are generalized in two respects: by including strange quarks and by going beyond the mean field level and adding the collision term. The exact expression involving collision integrals is replaced by the relaxation time approximation and numerical results are obtained.

\section{A. Derivation}

After studying a collisionless system, we extend our approach in order to include collisions. These can be incorporated by generalizing Eq. (2.2) to [6]

$$
\left(i \not \partial_{x}-m(x)\right) G^{<}(x, z)=\int d^{4} y\left(\Sigma^{c}(x, y) G^{<}(y, z)-\Sigma^{<}(x, y) G^{a}(y, z)\right),
$$

where the causal self energy $\Sigma^{c}$, the anticausal Green function $G^{a}$ and the self energy $\Sigma^{<}$appear on the right hand side. Since we also consider the production of strange particles, we have to extend the underlying dynamical model. The Lagrangian for the $S U_{f}(3)$ NJL model reads [1, 15]

$$
\begin{aligned}
\mathcal{L} & =\sum_{f=u, d, s} \bar{\psi}_{f}\left(i \not \partial-m_{0 f}\right) \psi_{f}+G \sum_{a=0}^{8}\left[\left(\bar{\psi} \lambda^{a} \psi\right)^{2}+\left(\bar{\psi} i \gamma_{5} \lambda^{a} \psi\right)^{2}\right] \\
& -K\left[\operatorname{det} \bar{\psi}\left(1+\gamma_{5}\right) \psi+\operatorname{det} \bar{\psi}\left(1-\gamma_{5}\right) \psi\right] .
\end{aligned}
$$


Equation (3.2) contains two coupling constants $G$ and $K$. The matrices $\lambda^{a}$ are the Gell-Mann matrices in flavor space with $\lambda^{0}=\sqrt{2 / 3}$. The Green function $G^{<}$ in the quasiparticle approximation is still diagonal in flavor space, but no longer independent of flavor:

$$
\begin{aligned}
G^{<}(x, p)=\frac{i \pi}{2 E_{f}(x, \vec{p})} \frac{\delta_{f f^{\prime}} \delta_{c c^{\prime}}}{N_{c}}\left(\not p+m_{f}(x)\right)\left[\delta\left(p_{0}-E_{f}(x, \vec{p})\right) n_{f}(x, \vec{p})\right. \\
\left.-\delta\left(p_{0}+E_{f}(x, \vec{p})\right)\left(2 N_{c}-n_{\bar{f}}(x,-\vec{p})\right)\right]
\end{aligned}
$$

where $n_{f}(x, \vec{p})$ is the density of quarks of flavor $f$ for $f=u, d, s$ and $E_{f}(x, \vec{p})=$ $\sqrt{\vec{p}^{2}+m_{f}^{2}(x)}$. The mean field derived from the Lagrangian (3.2) becomes 1, 15

$$
\begin{aligned}
& m_{f}(x)=m_{0 f}+ 4 G m_{f}(x) \int_{|\vec{p}|<\Lambda} \frac{d^{3} p}{(2 \pi)^{3}} \frac{2 N_{c}-n_{f}(x, \vec{p})-n_{\bar{f}}(x, \vec{p})}{E_{f}(x, \vec{p})} \\
&+ 2 K m_{f^{\prime}}(x) \int_{|\vec{p}|<\Lambda} \frac{d^{3} p}{(2 \pi)^{3}} \frac{2 N_{c}-n_{f^{\prime}}(x, \vec{p})-n_{\bar{f}^{\prime}}(x, \vec{p})}{E_{f^{\prime}}(x, \vec{p})} \\
& \times m_{f^{\prime \prime}}(x) \int_{|\vec{p}|<\Lambda} \frac{d^{3} p}{(2 \pi)^{3}} \frac{2 N_{c}-n_{f^{\prime \prime}}(x, \vec{p})-n_{\bar{f}^{\prime \prime}}(x, \vec{p})}{E_{f^{\prime \prime}}(x, \vec{p})}
\end{aligned}
$$

where $f, f^{\prime}$ and $f^{\prime \prime}$ are pairwise distinct flavors.

After performing a Wigner transformation and a gradient expansion of Eq. (3.1), inserting Eq. (3.3) and taking the real part of the resulting equation, one arrives at a Boltzmann like equation [4]

$$
\left(\partial_{t}+\vec{v}_{f} \vec{\partial}_{x}-\vec{\partial}_{x} E_{f} \vec{\partial}_{p}\right) n_{f}(x, \vec{p})=\sum_{f_{1}, f^{\prime}, f_{1}^{\prime}}\left(I_{f f_{1} \rightarrow f^{\prime} f_{1}^{\prime}}^{\text {coll }}+I_{f \bar{f}_{1} \rightarrow f^{\prime} \bar{f}_{1}^{\prime}}^{\text {coll }}\right)
$$

where $I_{f f_{1} \rightarrow f^{\prime} f_{1}^{\prime}}^{\text {coll }}$ and $I_{f \bar{f}_{1} \rightarrow f^{\prime} \bar{f}_{1}^{\prime}}^{\text {coll }}$ are the collision integrals due to quark-quark scattering $f f_{1} \rightarrow f^{\prime} f_{1}^{\prime}$ and quark-antiquark scattering $f \bar{f}_{1} \rightarrow f^{\prime} \bar{f}_{1}^{\prime}$, respectively. One has

$$
\begin{aligned}
& I_{f f_{1} \rightarrow f^{\prime} f_{1}^{\prime}}^{\text {coll }}=\frac{1}{2 E_{f}} \int d Q(2 \pi)^{4} \delta^{4}\left(p+p_{1}-p^{\prime}-p_{1}^{\prime}\right) \frac{1}{2} \overline{|\mathcal{M}|^{2}}{ }_{f f_{1} \rightarrow f^{\prime} f_{1}^{\prime}} \\
& \quad\left[n_{f^{\prime}}\left(x, \vec{p}^{\prime}\right) n_{f_{1}^{\prime}}\left(x, \vec{p}_{1}^{\prime}\right) \phi_{f}(x, \vec{p}) \phi_{f_{1}}\left(x, \vec{p}_{1}\right)-n_{f}(x, \vec{p}) n_{f_{1}}\left(x, \vec{p}_{1}\right) \phi_{f^{\prime}}\left(x, \vec{p}^{\prime}\right) \phi_{f_{1}^{\prime}}\left(x, \vec{p}_{1}^{\prime}\right)\right],
\end{aligned}
$$

with the statistical factor $1 / 2$ appearing in front of the squared transition amplitude. In Eq. (3.6) we have used the invariant integration volume

$$
d Q=\frac{d^{3} p_{1}}{(2 \pi)^{3} 2 E_{f_{1}}} \frac{d^{3} p^{\prime}}{(2 \pi)^{3} 2 E_{f^{\prime}}} \frac{d^{3} p_{1}^{\prime}}{(2 \pi)^{3} 2 E_{f_{1}^{\prime}}}
$$

and the blocking factors 


$$
\phi_{f}(x, \vec{p})=1-\frac{n_{f}(x, \vec{p})}{2 N_{c}}
$$

In contrast to Eq. (3.6), the collision integral due to quark-antiquark scattering does not contain the statistical factor. One has

$$
\begin{gathered}
I_{f \bar{f}_{1} \rightarrow f^{\prime} \bar{f}_{1}^{\prime}}^{\text {coll }}=\frac{1}{2 E_{f}} \int d Q(2 \pi)^{4} \delta^{4}\left(p+p_{1}-p^{\prime}-p_{1}^{\prime}\right){\overline{|\mathcal{M}|^{2}}}_{f \bar{f}_{1} \rightarrow f^{\prime} \bar{f}_{1}^{\prime}} \\
{\left[n_{f^{\prime}}\left(x, \vec{p}^{\prime}\right) n_{\bar{f}_{1}^{\prime}}\left(x, \vec{p}_{1}^{\prime}\right) \phi_{f}(x, \vec{p}) \phi_{\bar{f}_{1}}\left(x, \vec{p}_{1}\right)-n_{f}(x, \vec{p}) n_{\bar{f}_{1}}\left(x, \vec{p}_{1}\right) \phi_{f^{\prime}}\left(x, \vec{p}^{\prime}\right) \phi_{\bar{f}_{1}^{\prime}}\left(x, \vec{p}_{1}^{\prime}\right)\right] .}
\end{gathered}
$$

Note that, in terms of the differential cross section, one has 16]

$$
\frac{1}{2 E_{f}} d Q(2 \pi)^{4} \delta^{4}\left(p+p_{1}-p^{\prime}-p_{1}^{\prime}\right) \kappa \overline{|\mathcal{M}|^{2}}=\frac{d^{3} p_{1}}{(2 \pi)^{3}} d \Omega v_{\mathrm{rel}} \frac{d \sigma}{d \Omega}
$$

where $\kappa$ is a statistical factor which equals $1 / 2$ for the elastic scattering of identical particles and 1 for all other processes. Equations (3.5), (3.6) and (3.9) form the basis of our following investigations.

\section{B. Relaxation Time Approximation}

The collision integrals in Eq. (3.5) contain the differential cross sections $d \sigma / d \Omega$ for elastic scattering processes like $u u \rightarrow u u$ and inelastic ones like $u \bar{u} \rightarrow s \bar{s}$. The scattering amplitude $\mathcal{M}$ in Eqs. (3.6), (3.9) depends on the selfconsistent masses $m_{f}(x)$ and on the actual densities $n_{f}(x, \vec{p})$ via the Pauli factors [5, 15]. Thus $\mathcal{M}$ has to be recalculated at every space-time point of the expansion. This is a formidable numerical task and goes beyond the scope of this paper. Therefore we use the relaxation time approximation. Our ansatz for the transport equation is

$$
\begin{aligned}
& \left(\partial_{t}+\vec{v}_{q} \vec{\partial}_{x}-\vec{\partial}_{x} E_{q} \vec{\partial}_{p}\right) n_{q}(x, \vec{p})=\frac{\tilde{n}_{q}(x, \vec{p})-n_{q}(x, \vec{p})}{\tau_{q q}(x, \vec{p})}-\frac{n_{q}(x, \vec{p})}{\tau_{q s}(x, \vec{p})}+G_{q}(x, \vec{p}) \\
& \left(\partial_{t}+\vec{v}_{s} \vec{\partial}_{x}-\vec{\partial}_{x} E_{s} \vec{\partial}_{p}\right) n_{s}(x, \vec{p})=\frac{\tilde{n}_{s}(x, \vec{p})-n_{s}(x, \vec{p})}{\tau_{s s}(x, \vec{p})}-\frac{n_{s}(x, \vec{p})}{\tau_{s q}(x, \vec{p})}+G_{s}(x, \vec{p})
\end{aligned}
$$

As in Section प1, we set $n_{u}(x, \vec{p})=n_{d}(x, \vec{p})=n_{q}(x, \vec{p}) / 2$ and also $n_{f}(x, \vec{p})=n_{\bar{f}}(x, \vec{p})$. The first term on the right hand side of Eq. (3.11a) describes gain and loss terms due to the elastic scattering of light quarks. In this term, $\tilde{n}_{q}(x, \vec{p})$ denotes an effective equilibrium distribution function to be detailled below. The second term describes the loss of light quarks due to the processes $u \bar{u} \rightarrow s \bar{s}$ and $d \bar{d} \rightarrow s \bar{s}$. The 
last term models the gain of light quarks from strange quarks due to the reverse processes. Its concrete form will also be given below. The terms on the right hand side of Eq. (3.11b) have similar origins, with light and strange quarks being suitably exchanged. The relaxation times $\tau_{q q}, \tau_{s s}, \tau_{q s}$ and $\tau_{s q}$ are calculated from the equilibrium cross sections [5]. They will be given in Section [110.

The effective densities $\tilde{n}_{q}(x, \vec{p})$ and $\tilde{n}_{s}(x, \vec{p})$ are chosen to have the form

$$
\begin{aligned}
& \tilde{n}_{q}(x, \vec{p})=\frac{4 N_{c}}{\exp \left(p_{\mu} u_{q}^{\mu}(x) / T(x)\right)+1} \\
& \tilde{n}_{s}(x, \vec{p})=\zeta(x) \frac{2 N_{c}}{\exp \left(p_{\mu} u_{s}^{\mu}(x) / T(x)\right)+1},
\end{aligned}
$$

where $u_{f}^{\mu}(x)$ is the collective velocity of the fluid component with flavor $f$ at spacetime point $x, T(x)$ an effective flavor independent temperature and $\zeta(x)$ a factor, which measures the amount of chemical equilibrium for the strange quarks. The velocity $u_{f}^{\mu}(x)$ can be calculated from the numerical solution $n_{f}(x, \vec{p})$ at every time slice,

$$
u_{f}^{\mu}(x)=\int \frac{d^{3} p}{(2 \pi)^{3}} \frac{p^{\mu}}{E} n_{f}(x, \vec{p}) / \int \frac{d^{3} p}{(2 \pi)^{3}} n_{f}(x, \vec{p})
$$

so that $T(x)$ and $\zeta(x)$ remain to be determined.

The requirement of energy conservation is used to fix the effective temperature $T(x)$ and the density factor $\zeta(x)$. After introducing a potential energy which, as for the $S U_{f}(2)$ case, compensates the change of the kinetic energy due to the mean field, one obtains

$$
\begin{aligned}
\frac{1}{2} \frac{d \mathcal{E}_{\mathrm{tot}}}{d t} & =\int \frac{d^{3} x d^{3} p}{(2 \pi)^{3}} \frac{E_{q}(x, \vec{p})}{\tau_{q q}(x, \vec{p})}\left(\tilde{n}_{q}(x, \vec{p})-n_{q}(x, \vec{p})\right) \\
& +\int \frac{d^{3} x d^{3} p}{(2 \pi)^{3}} \frac{E_{s}(x, \vec{p})}{\tau_{s s}(x, \vec{p})}\left(\tilde{n}_{s}(x, \vec{p})-n_{s}(x, \vec{p})\right) \\
& +\int \frac{d^{3} x d^{3} p}{(2 \pi)^{3}}\left(E_{s}(x, \vec{p}) G_{s}(x, \vec{p})-\frac{E_{q}(x, \vec{p}) n_{q}(x, \vec{p})}{\tau_{q s}(x, \vec{p})}\right) \\
& +\int \frac{d^{3} x d^{3} p}{(2 \pi)^{3}}\left(E_{q}(x, \vec{p}) G_{q}(x, \vec{p})-\frac{E_{s}(x, \vec{p}) n_{s}(x, \vec{p})}{\tau_{s q}(x, \vec{p})}\right)
\end{aligned}
$$

for the change of the total energy due to the collisional part of Eqs. (3.11). The factor $1 / 2$ at the left hand side accounts for the antiparticle degree of freedom. Note that for the Lagrangian (3.2) an effective potential, as it was given in Eq. (2.18) for 
the $S U(2)$ case, exists in a mathematical sense, however, due to the six fermion couplings in the 't Hooft determinant, it is not possible to compute it analytically. For the exact Boltzmann equation (3.5), each of the integrals on the right hand side of Eq. (3.14) vanishes individually, even if the spatial integrations are dropped. We adopt this stronger constraint also for Eq. (3.14), and have the two conditions

$$
\begin{aligned}
& \int \frac{d^{3} p}{(2 \pi)^{3}} \frac{E_{q}(x, \vec{p})}{\tau_{q q}(x, \vec{p})}\left(\tilde{n}_{q}(x, \vec{p})-n_{q}(x, \vec{p})\right)=0 \\
& \int \frac{d^{3} p}{(2 \pi)^{3}} \frac{E_{s}(x, \vec{p})}{\tau_{s s}(x, \vec{p})}\left(\tilde{n}_{s}(x, \vec{p})-n_{s}(x, \vec{p})\right)=0
\end{aligned}
$$

to determine $T(x)$ and $\zeta(x)$ at each space-time point. In order to fulfill the remaining constraints

$$
\begin{aligned}
& \int \frac{d^{3} p}{(2 \pi)^{3}}\left(E_{s}(x, \vec{p}) G_{s}(x, \vec{p})-\frac{E_{q}(x, \vec{p}) n_{q}(x, \vec{p})}{\tau_{q s}(x, \vec{p})}\right)=0 \\
& \int \frac{d^{3} p}{(2 \pi)^{3}}\left(E_{q}(x, \vec{p}) G_{q}(x, \vec{p})-\frac{E_{s}(x, \vec{p}) n_{s}(x, \vec{p})}{\tau_{s q}(x, \vec{p})}\right)=0
\end{aligned}
$$

we make the ansatz that the gain terms $G_{q}$ and $G_{s}$ are proportional to the densities in local thermal equilibrium:

$$
\begin{aligned}
G_{q}(x, \vec{p}) & =\gamma_{q}(x) \frac{\tilde{n}_{q}(x, \vec{p})}{\tau_{q s}(x, \vec{p})} \\
G_{s}(x, \vec{p}) & =\gamma_{s}(x) \frac{\tilde{n}_{s}(x, \vec{p}) / \zeta(x)}{\tau_{s q}(x, \vec{p})} .
\end{aligned}
$$

The factors $\gamma_{q}(x)$ and $\gamma_{s}(x)$ are determined by solving Eqs. (3.16):

$$
\begin{aligned}
& \gamma_{q}(x)=\int \frac{d^{3} p}{(2 \pi)^{3}} \frac{E_{s}(x, \vec{p}) n_{s}(x, \vec{p})}{\tau_{s q}(x, \vec{p})} / \int \frac{d^{3} p}{(2 \pi)^{3}} \frac{E_{q}(x, \vec{p}) \tilde{n}_{q}(x, \vec{p})}{\tau_{q s}(x, \vec{p})} \\
& \gamma_{s}(x)=\int \frac{d^{3} p}{(2 \pi)^{3}} \frac{E_{q}(x, \vec{p}) n_{q}(x, \vec{p})}{\tau_{q s}(x, \vec{p})} / \int \frac{d^{3} p}{(2 \pi)^{3}} \frac{E_{s}(x, \vec{p}) \tilde{n}_{s}(x, \vec{p}) / \zeta(x)}{\tau_{s q}(x, \vec{p})} .
\end{aligned}
$$

With this form of the gain terms, the collision term vanishes in local thermal equilibrium.

\section{Relaxation Times}

The basic assumption of the relaxation time approximation is that the system is close to equilibrium and that the right hand side of the Boltzmann equation is 
only linear in the difference $n_{f}-\tilde{n}_{f}$. Therefore the relaxation times have to be evaluated with the equilibrium densities and masses and depend only on the local temperature.

The collision term on the left hand side of Eq. (3.5) involves a sum over all possible scattering processes. The task of computing the relaxation times for Eq. (3.11) is thus twofold: firstly one has to define the relaxation time for one single process and afterwards add up the contributions of several processes in order to obtain Eqs. (3.11).

In order to define the the relaxation times, we go back to Eq. (3.5) and write it in the form

$$
\left(\partial_{t}+\vec{v}_{f} \vec{\partial}_{x}-\vec{\partial}_{x} E_{f} \vec{\partial}_{p}\right) n_{f}(x, \vec{p})=\sum_{P}\left(I_{P, \text { gain }}^{f}-I_{P, \text { loss }}^{f}\right)
$$

where the sum runs over all possible processes and $I_{P \text {,gain }}^{f}$ and $I_{P, \text { loss }}^{f}$ are the respective gain and loss terms due to process $P$. We focus on one generic process $P: f f_{1} \leftrightarrow$ $f^{\prime} f_{1}^{\prime}$. The loss term due to this process is given by

$$
I_{P, \text { loss }}^{f}=\int \frac{d^{3} p_{1}}{(2 \pi)^{3}} d \Omega v_{\text {rel }} \frac{d \sigma_{P}}{d \Omega} n_{f} n_{f_{1}} \phi_{f^{\prime}} \phi_{f_{1}^{\prime}}
$$

By comparing this to the ansatz (3.11), one immediately obtains the relaxation time for this process

$$
\frac{1}{\tau_{P}(x, \vec{p})}=\int \frac{d^{3} p_{1}}{(2 \pi)^{3}} d \Omega v_{\mathrm{rel}} \frac{d \sigma_{P}}{d \Omega} n_{f_{1}} \phi_{f^{\prime}} \phi_{f_{1}^{\prime}}
$$

In order to evaluate this expression, we go to the rest frame of the plasma and approximate the differential cross section by its equilibrium value at the local temperature $T(x)$, the density of flavor $f_{1}$ by

$$
n_{f_{1}}\left(T(x), p_{1}\right)=\frac{2 N_{c}}{\exp \left[E_{f_{1}}\left(T(x), p_{1}\right) / T(x)\right]+1}
$$

where $E_{f_{1}}\left(T(x), p_{1}\right)$ is computed using the equilibrium mass corresponding to the effective temperature $T(x)$. The blocking factors are replaced by

$$
\phi_{f^{\prime}}(T(x), s)=1-\frac{1}{\exp \left[E_{f^{\prime}}^{\mathrm{cm}}(T(x), s) / T(x)\right]+1},
$$

where $E_{f^{\prime}}^{\mathrm{cm}}(T(x), s)$ is the energy for the participant $f^{\prime}$ in the centre of mass frame, which can be expressed as a function of the masses and the Mandelstam variable $s$. These replacements lead to 


$$
\frac{1}{\tau_{P}\left(T(x), p_{f}\right)}=\int \frac{d^{3} p_{1}}{(2 \pi)^{3}} v_{\mathrm{rel}} \sigma_{P, \mathrm{eff}}(T(x), s) n_{f_{1}}\left(T(x), p_{1}\right) .
$$

The effective cross section in Eq. (3.24) is defined by

$$
\sigma_{P, \mathrm{eff}}(T(x), s)=\phi_{f^{\prime}}(T(x), s) \phi_{f_{1}^{\prime}}(T(x), s) \int d \Omega \frac{d \sigma_{P}}{d \Omega}(T(x), s, t) .
$$

By introducing the Mandelstam variable $s$ as integration variable, Eq. (3.24) can be reduced to

$$
\begin{gathered}
\frac{1}{\tau_{P}\left(T(x), p_{f}\right)}=\frac{1}{16 \pi^{2}} \frac{1}{E_{f} p_{f}} \int_{\left(m_{f}+m_{f_{1}}\right)^{2}}^{\infty} d s \sqrt{\left[s-\left(m_{f}+m_{f_{1}}\right)^{2}\right]\left[s-\left(m_{f}-m_{f_{1}}\right)^{2}\right]} \\
\quad \times \sigma_{P, \text { eff }}(T(x), s) \omega_{f f_{1}}\left(T(x), s, E_{f}\right)
\end{gathered}
$$

where

$\omega_{f f_{1}}\left(T(x), s, E_{f}\right)=\int d E_{f_{1}} n_{f_{1}}\left(T(x), p_{1}\right) \Theta\left[\left(2 p_{f} p_{f_{1}}\right)^{2}-\left(s-m_{f}^{2}-m_{f_{1}}^{2}-2 E_{f} E_{f_{1}}\right)^{2}\right]$

is a weight function, which can be computed analytically for $n_{f_{1}}$ given by Eq. (3.22). The temperature dependent cross sections entering Eq. (3.26) are taken from Ref. [5]. Note that $\tau_{P}$ in the rest system of the plasma is a function of $T(x)$ and $p_{f}$ only. It can thus be easily obtained via table lookup. In the lab system, we compute $\tau_{P}(x, \vec{p})$ by applying a Lorentz boost to Eq. (3.26).

In order to relate the relaxation time for a specific process to those needed in Eq. (3.11), we consider again the loss term of the exact equation (3.5). To be specific, we set $f=u$. Then the exact loss term can be decomposed into the contributions

$$
\begin{aligned}
\sum_{P} I_{P, \mathrm{loss}}^{u} & =I_{u u \rightarrow u u, \mathrm{loss}}^{u}+I_{u d \rightarrow u d, \mathrm{loss}}^{u}+I_{u s \rightarrow u s, \mathrm{loss}}^{u} \\
& +I_{u \bar{u} \rightarrow u \bar{u}, \mathrm{loss}}^{u}+I_{u \bar{d} \rightarrow u \bar{d}, \mathrm{loss}}^{u}+I_{u \bar{s} \rightarrow u \bar{s}, \mathrm{loss}}^{u} \\
& +I_{u \bar{u} \rightarrow d \bar{d}, \mathrm{loss}}^{u}+I_{u \bar{u} \rightarrow s \bar{s}, \mathrm{loss}}^{u}
\end{aligned}
$$

Only the last two of these contributions correspond to inelastic processes. However, we have considered $u$ and $d$ to be degenerate in Eq. (3.11), so that we have to include also the contribution of $u \bar{u} \rightarrow d \bar{d}$ to the elastic relaxation time $\tau_{q q}$ of Eq. (3.11). Thus one has

$$
\begin{aligned}
\frac{1}{\tau_{q q}} & =\frac{1}{\tau_{u u \rightarrow u u}}+\frac{1}{\tau_{u d \rightarrow u d}}+\frac{\zeta}{\tau_{u s \rightarrow u s}}+\frac{1}{\tau_{u \bar{u} \rightarrow u \bar{u}}}+\frac{1}{\tau_{u \bar{d} \rightarrow u \bar{d}}}+\frac{\zeta}{\tau_{u \bar{s} \rightarrow u \bar{s}}}+\frac{1}{\tau_{u \bar{u} \rightarrow d \bar{d}}} \\
\frac{1}{\tau_{q s}} & =\frac{1}{\tau_{u \bar{u} \rightarrow s \bar{s}}}
\end{aligned}
$$


where a factor $\zeta$ has been multiplied to the contributions of the processes us $\rightarrow$ us and $u \bar{s} \rightarrow u \bar{s}$ in order to relate Eq. (3.22) to Eq. (3.12b). By considering the loss term for the strange quark, one obtains in an analogous fashion

$$
\begin{aligned}
\frac{1}{\tau_{s s}} & =\frac{\zeta}{\tau_{s s \rightarrow s s}}+\frac{2}{\tau_{s u \rightarrow s u}}+\frac{\zeta}{\tau_{s \bar{s} \rightarrow s \bar{s}}}+\frac{2}{\tau_{s \bar{u} \rightarrow s \bar{u}}} \\
\frac{1}{\tau_{s q}} & =\frac{2 \zeta}{\tau_{s \bar{s} \rightarrow u \bar{u}}}
\end{aligned}
$$

where the factor 2 in the contributions of $s u \rightarrow s u, s \bar{u} \rightarrow s \bar{u}$ and $s \bar{s} \rightarrow u \bar{u}$ accounts for the fact that in all these processes $u$ can be replaced by $d$.

In Fig. 6, we show the resulting inverse relaxation times $\tau^{-1}$ for the elastic scattering of light quarks (solid line), the elastic scattering of strange quarks (dashed line) and for the production of strange quarks (dotted line) as a function of temperature and for a momentum $p=200 \mathrm{MeV}$. While $\tau_{q q}^{-1}$ and $\tau_{s s}^{-1}$ are approximately equal, $\tau_{q s}^{-1}$ is around two orders of magnitude lower than $\tau_{q q}^{-1}$ for this value of $p$. The main reason for this is the threshold for the process $q \bar{q} \rightarrow s \bar{s}$, which makes this process improbable at low momenta. The ratio between $\tau_{q q}^{-1}$ and $\tau_{q s}^{-1}$ decreases for increasing momenta. At low temperatures, $\tau^{-1}$ goes to zero rapidly, mainly because of the density factor in Eq. (3.24). The solid, dashed and dotted curves of Fig. 6 are calculated using temperature dependent quark masses and the temperature dependent cross sections given in Ref. [5]. These are to be contrasted with the dot-dashed line, which gives $\tau_{q q}^{-1}$ from a calculation, where the masses have been set to the current quark masses and the cross sections have been calculated in the Born approximation [14], which does not include any medium dependence. At high temperatures, this procedure results in an inverse relaxation time, which is approximately a factor 3.5 smaller, whereas the falloff at low temperatures is weaker due to the lower masses.

In Fig. 17, $\tau_{q q}^{-1}$ is given as a function of temperature for $p=0$ (solid line) and $p=200 \mathrm{MeV}$ (dashed line). One notes that the solid line displays a pronounced maximum at $T \approx 230 \mathrm{MeV}$. It originates from a divergence of the scattering length, which occurs for the quark-antiquark scattering processes at the Mott temperature, where the pion becomes unbound [5, 17]. This phenomenon is related to critical scattering. The Mott temperature is ca. $210 \mathrm{MeV}$ for our parameter set. As to be expected, the Born calculation for $p=0$, which is given by the dotted line of Fig. 7 , displays no structure at this temperature. 


\section{Numerical Results}

\section{Densities, Quark Masses, Spectra and Strangeness Yield}

The transport equations (3.11) are solved using an obvious generalization of the numerical methods outlined in Section [ID. In this subsection we show results of a calculation with the initial condition

$$
\begin{aligned}
& n_{q}(x, \vec{p})=\exp \left(-\frac{r^{2}}{2 r_{0}^{2}}\right) \frac{4 N_{c}}{\exp \left(\sqrt{\vec{p}^{2}+m_{i}^{2}} / T_{0}\right)+1} \\
& n_{s}(x, \vec{p})=0 .
\end{aligned}
$$

Comparing the form of Eq. 3.31a) with Eq. (2.30) there is only one difference, namely the absence of a cutoff in momentum space: While Eq. (2.30) contains a smooth cutoff, we now continue the Fermi shape of the distribution up to the grid boundary $p_{\max }$, which in our standard parameter set is set to $p_{\max }=\Lambda$. This modification is necessary if one wants to determine effective temperatures from an exponential fit to the particle spectra. The disadvantage of this modification is that the total particle number and the total energy are no longer exactly conserved. We will discuss the magnitude of these effects later.

The standard values of the parameters are the same as used in Section IIE: $r_{0}=3 \mathrm{fm}$ and $T_{0}=240 \mathrm{MeV}$; the initialization mass $m_{i}$ is taken to be a solution of Eq. (3.4) at $r=0$. The NJL model parameters for all $S U_{f}(3)$ calculations are taken from Ref. [15 and are $m_{0 q}=5.5 \mathrm{MeV}, m_{0 s}=140.7 \mathrm{MeV}, G \Lambda^{2}=1.835$, $K \Lambda^{5}=12.36$ and $\Lambda=602.3 \mathrm{MeV}$.

The time dependence of the light quark density is shown in Fig. 8. This figure should be compared with the same quantity calculated for the Vlasov equation, shown in Fig. 2. The time steps coincide in both figures. The behaviour of the light quark densities is rather similar. The time variation for the density of strange quarks is shown in Fig. 9. When comparing it to Fig. 8 for the light quarks, one should first note the different scale on the ordinate (about a factor of 30 down), and secondly the initial condition $n_{s}(t=0, \vec{x}, \vec{p})=0$. The density rises due to strange quark production until $t=4 \mathrm{fm} / c$, after which time the rarefaction due to flow overcomes the production and the density drops. 
The light and strange quark masses are shown in Fig. 10 as a function of time. For the light quark mass one obtains essentially the same behaviour as observed in the $S U(2)$ case shown in Fig. 3. At $t=0$ the light quark mass amounts to $m_{q}=58 \mathrm{MeV}$ in the centre and rises to the vacuum value $m_{q}=368 \mathrm{MeV}$ in the outer regions. As time progresses, the masses increase everywhere to this value. For the strange quarks, on the other hand, one does not observe strong variations of the constituent mass. This different behaviour in the space-time behavior of $m_{q}(\vec{x}, t)$ and $m_{s}(\vec{x}, t)$ is significant. While the difference in the vacuum values $m_{s}-m_{q}=182 \mathrm{MeV}$ compares well with the mass difference between lambda and proton, $m_{\Lambda}-m_{p}=177 \mathrm{MeV}$, the in medium corrections in the plasma are large for the light quarks and small for the strange quarks. In the NJL model the medium effects on the masses can be traced to the Pauli-blocking, essentially. The Pauli-blocking for the strange quarks is small because of the low density of this kind of quarks. At $t=0$, the strange quark density vanishes and only the term proportional to $K$ in Eq. (3.4) gives corrections to the vacuum mass. This leads to a value of $m_{s}=465 \mathrm{MeV}$ in the centre and $m_{s}=550 \mathrm{MeV}$ in the outer regions. At later times, the term proportional to $G$ also gives medium corrections. However, these are not sufficient to lower the strange quark mass substantially in the central region. With increasing time, also the strange quark mass evolves to its vacuum expectation value everywhere, as expected. The large mass difference of the in medium masses suppresses the production of strange quarks in our calculation - and possibly in nature.

In Fig. 11, we show the "observed" (for $t \rightarrow \infty$ ) distributions of particles in momentum space, $d \mathcal{N} / d^{3} p$, as a function of the particle energy. An exponential fit is applied to the high energy part of the spectra (dashed lines). The effective slope temperatures of these fits for the light and strange quarks are different: $T_{q}=185 \mathrm{MeV}$ for light quarks and $T_{s}=164 \mathrm{MeV}$ for the strange ones. The calculated spectra show an enhancement at low energies compared to the exponential fit. This enhancement arises from the mean field, as has already been explained in Section IIE. We comment on the difference $T_{q}-T_{s}$ in Section IV.

We find for the strangeness yield, defined as the ratio of the number of strange quarks to that of light quarks at $t=\infty$, a value of $\mathcal{N}_{s, t=\infty} / \mathcal{N}_{q, t=\infty}=2.2 \%$. This value is rather low and is mainly determined by incomplete chemical equilibration, as we will show in in Section IV. 


\section{Dependencies on the Grid Settings and Numerical Accuracy}

In order to see the reliability of the results and the stability with respect to the numerical scheme, we have investigated the dependence of the particle multiplicities and the spectra on the grid extension. The results of the various runs are compiled in Table $\mathbb{\text { I. }}$. The calculated multiplicities of the light and strange quarks with the standard parameters described above are given in the row labeled (a). One has $\mathcal{N}_{q, t=0}=343.2$ in the initial state and $\mathcal{N}_{q, t=\infty}=365.6$ and $\mathcal{N}_{s, t=\infty}=8.1$ in the final state. This corresponds to a relative yield of $\mathcal{N}_{s, t=\infty} / \mathcal{N}_{q, t=\infty}=2.2 \%$. We observe an increase of the total multiplicity from $\left(\mathcal{N}_{q}+\mathcal{N}_{s}\right)_{t=0}=343.2$ to $\left(\mathcal{N}_{q}+\mathcal{N}_{s}\right)_{t=\infty}=$ 373.7. However, $\mathcal{N}_{q}+\mathcal{N}_{s}$ should be a constant of motion for the exact Boltzmann equation $(3.5)$.

The possible reasons for the increase of the increase are the following: (i) numerical inaccuracies, (ii) the relaxation time ansatz, which, however, should lead to a decrease of the particle number due to the dropping temperature and (iii) the extension of the grid in momentum space. The last effect can be understood as follows: In the calculation related to row (a) of Table 目, the grid has maximal momentum $p_{\max }=\Lambda=602 \mathrm{MeV}$ and the initial "thermal" distribution of Eqs. (3.31) is cut off at this value. Calculating also the particles from Eq. (3.31) with $p \geq p_{\max }$, we see that our grid includes only $40 \%$ of all particles explicitly. Nevertheless, due to the open boundary conditions which are chosen in Eq. (2.29), i. e. smoothness at $p=p_{\max }$, the numerical procedure assumes that the particle density can be extrapolated analytically beyond the grid boundary and thus "recognizes" the remaining particles implicitly, at least as far as the derivative terms of the transport equation are concerned. Since the mean field tends to slow down particles, it is possible that particles with $p \geq p_{\max }$, which are not counted at $t=0$, appear inside the grid at later times, and thus the multiplicity increases.

In order to eliminate the influence of the finite grid extension in momentum space, two other settings have been used: firstly, the grid is extended to include momenta up to $p_{\max }=3 \Lambda$. In this calculation, all momentum integrals have been extended to $p=$ $p_{\text {max }}$, except those occurring in the gap equation (3.4). The resulting multiplicities are given in row (b) of Table $\mathbb{\text { I. }}$. Compared to the $8.9 \%$ increase observed in the previous calculation, one now obtains a increase of $5.8 \%$ of the particle number, 
which is comparable with the Vlasov result. The fraction of strange particles in this calculation is $3.9 \%$, compared to $2.2 \%$ for calculation (a).

In a second investigation a smooth cutoff for the high momentum region is introduced in the initial conditions, as is done for the Vlasov equation in Eq. (2.30). In this calculation, the effective distribution functions of Eqs. (3.12) are consistently cut off by the same factor. This procedure is also consistent with the requirement of energy conservation, as has been explained in Section IIC, and with the nature of the NJL model as a low energy theory. The multiplicities for this calculation are given in row (c) of Table $\mathbb{1}$. We observe a 1.3\% decrease of the particle number, which means that the particle number is in good approximation conserved. The strangeness content in the final state amounts to $2.3 \%$.

The light quark spectra of calculations (a), (b) and (c) are shown in Fig. 12. The data for the calculation (a) (solid line) agree with those for calculation (b) (dashed line). The data for the calculation (c) are given by the dotted line. Although calculation (c) agrees reasonably with (a) and (b) in the low momentum region (as it should), the momentum cutoff factor distorts the high momentum part completely. It is thus impossible to fit a reasonable slope temperature to this curve.

While the agreement of the spectra is good for light quarks, this is not the case for strange quarks, as can be seen from Fig. 13. Calculation (b), i. e. $p_{\max }=3 \Lambda$, gives a twice higher value for $d \mathcal{N}_{s} / d^{3} p$ as compared to calculation (a) and (c), i. e. $p_{\max }=\Lambda$, since more light quarks with high energy are available for the production of strange quarks.

This may be the appropriate place to report the numerical effort and accuracy. The use of a partial differential equation in four variables for the expansion of a spherical system in phase space is limited by the use of computer memory and CPU time. For a $50^{3}$ grid, our program uses ca. 3 MByte memory and 20 seconds of CPU time per time slice on a Sparc 20 workstation. The grid size as well as the number of time slices has to be increased when e.g. increasing the radius $r_{0}$ of the fireball, so that the above numbers have to be scaled accordingly. Most of the CPU time is consumed by the determination of the effective temperature. All indicators point to an overall numerical accuracy of a few percent of our results. 


\section{Dependencies on Medium Effects}

The inclusion of medium effects in the collision terms is an important and new aspect of our calculation. Indeed, the medium effects are large, both for the relaxation times shown in Fig. [7, as well as for the quark masses shown in Fig. 10. It is thus important to investigate their influence on the final spectra. Our results are compiled in Table [1], where row (a) relates to the standard calculation. The medium effects are studied in two ways: by keeping or switching off the mean field and by replacing the cross sections in the relaxation times by their Born approximation 14] values. All other parameters correspond to the standard set. Multiplicities and slope temperatures for these runs are given in rows (d) and (e) of Table II], respectively. While the results of calculation (d) are similar to those of calculation (a), calculation (e), i. e. the one without mean field, gives completely different results for multiplicities and slope temperatures. This can also be seen in the light quark spectra given in Fig. 14 where the results of calculations (a), (d) and (e) are shown by the solid, dashed and dotted line, respectively. While (a) and (d) give almost identical results, calculation (e) differs substantially. Firstly, the energy range is shifted to smaller energies due to the small quark mass. Secondly, the spectrum is flatter, indicating a higher slope temperature. Thirdly, calculation (e) shows a deficiency of particles at low energies, instead of an enhancement like (a) and (d). This can be traced back to the fact that there is no mean field any longer, which shifts particles into this region.

We have also investigated, whether the maximum in the temperature dependence of the relaxation times in Fig. 7, i. e. critical scattering, has some effect on the final spectra. To this purpose we have artificially set $\tau(p, T)=\tau(p=200 \mathrm{MeV}, T)$ for $p<200 \mathrm{MeV}$ and have compared the result with the full equation. The resulting spectra agree with those of Fig. 11 within the accuracy of our calculation.

We conclude that (i) medium effects in the collision terms have small effects on the shape of the final spectra but they considerably change the strangeness yield (here by $35 \%$ ) and (ii) the medium effects in the mean field parts lead to significant effects in the spectra, especially in the low energy part. 


\section{STRANGENESS PRODUCTION}

We discuss the variation of the strangeness yield with the radius $r_{0}$ of the initial fireball. The observables of the final state are the slope temperatures $T_{q}$ and $T_{s}$ for the quarks and the strangeness yields. We have gradually increased the initial radius from $r_{0}=1 \mathrm{fm}$ to $r_{0}=7 \mathrm{fm}$ while the other parameters of Eq. (3.31) are kept fixed. The resulting values for the slope temperatures $T_{q}$ and $T_{s}$ and the strangeness yield $\mathcal{N}_{s} / \mathcal{N}_{q}$ are given in Figs. 15 and 16. While the slope temperature for light quarks hardly changes with the system size, the slope temperature for the strange quarks rises and approaches $T_{q}$ from below.

The independence of $T_{q}$ on $r_{0}$ corresponds to the independence of the hadronic slope temperatures on the projectile and target size, which has been reported in Ref. [18]. The fact that $T_{s}$ stays below $T_{q}$ seems to be in contradiction with experimental data [19] and hydrodynamical models [20]. These models claim that the slope temperatures scale with the particle mass like $T=T_{0}+m\left\langle v_{\perp}\right\rangle^{2}$, where $\left\langle v_{\perp}\right\rangle$ is the mean transverse expansion velocity. One has to keep in mind, however, that this theoretical result is significantly influenced by the cylindrical geometry of the colliding nuclei and by the presence of transverse flow. These features are not present in our calculation.

The increase of $T_{s}$ with the initial radius $r_{0}$, which can be seen in Fig. 15, is essentially an effect of thermal equilibration. For small initial size, where there is negligible thermal equilibration, one observes the "temperature" of the strange quarks directly from the creation process. Its low value can be understood qualitatively by assuming that the mean energy per particle $E=m_{q}+3 / 2 T_{q}=m_{s}+3 / 2 T_{s}$ is conserved during the creation of a strange quark pair. One thus obtains $T_{s}=T_{q}-2 / 3\left(m_{s}-m_{q}\right)$ immediately after the pair creation. Subsequent elastic collisions, as they will occur more frequently in the larger systems, result in $T_{s}$ approaching $T_{q}$. This is indeed observed in Fig. 15.

According to Fig. 16, the strangeness yield grows with the radius of the initial fireball. This behaviour of the curve can be understood using the following simple analytical model. We make the ansatz

$$
\frac{d \rho_{s}(\vec{x}, t)}{d t}+\operatorname{div}\left[\vec{v} \rho_{s}(\vec{x}, t)\right]=\alpha\left[\rho_{q}^{2}(\vec{x}, t)-\kappa \rho_{s}^{2}(\vec{x}, t)\right]
$$

for the space-time evolution of the strange quark density. In Eq. (4.1), the densities 
are parametrized by

$$
\begin{aligned}
& \rho_{q}(\vec{x}, t)=\frac{\mathcal{N}_{q}}{\frac{4 \pi}{3} r_{q}^{3}(t)} \Theta\left(r_{q}(t)-|\vec{x}|\right) \\
& \rho_{s}(\vec{x}, t)=\frac{\mathcal{N}_{s}(t)}{\frac{4 \pi}{3} r_{s}^{3}(t)} \Theta\left(r_{s}(t)-|\vec{x}|\right) .
\end{aligned}
$$

For the radius of the light quark system, we make the ansatz

$$
r_{q}^{2}(t)=r_{0}^{2}+v^{2} t^{2}
$$

with a constant velocity $v$. Equation (4.3) is motivated by the exact solution of the Vlasov equation in the noninteracting case. We assume that the radius of the strange quark system behaves similarly, however, is smaller by a factor $z$ :

$$
r_{s}(t)=z r_{q}(t)
$$

This factor accounts for the fact that only in the interior of the fireball the density is large enough to produce particles and is also born out numerically by comparing Figs. 8 and 9. With the parametrizations (4.2)-(4.4) at hand, Eq. (4.1) can be solved analytically to give

$$
\frac{\mathcal{N}_{s}(t)}{\mathcal{N}_{q}}=\sqrt{\frac{z^{3}}{\kappa}} \tanh \left(\frac{3 \alpha \sqrt{\kappa} \mathcal{N}_{q}}{4 \pi r_{0}^{2}} \frac{t}{\sqrt{r_{0}^{2}+v^{2} t^{2}}}\right) .
$$

In the limit $t \rightarrow \infty$, one thus has

$$
\frac{\mathcal{N}_{s}}{\mathcal{N}_{q}}=\sqrt{\frac{z^{3}}{\kappa}} \tanh \left(\frac{3 \alpha \sqrt{\kappa} \mathcal{N}_{q}}{4 \pi r_{0}^{2} v}\right) .
$$

Since $\mathcal{N}_{q} \sim r_{0}^{3}$, Eq. (4.6) predicts a dependence on the initial radius as

$$
\frac{\mathcal{N}_{s}}{\mathcal{N}_{q}} \sim \tanh \left(a r_{0}\right) .
$$

Figure 16 shows, that this behaviour is indeed fulfilled in our calculation. The fit shown in Fig. 16 (solid line) has the parameters

$$
\frac{\mathcal{N}_{s}}{\mathcal{N}_{q}}=0.065 \tanh \left(0.12 r_{0}\right) .
$$

The factor $6.5 \%$ in front of the hyperbolic tangent is the equilibrium value for an infinite system. A similar dependence like Eq. (4.7), however with different fit 
coefficients, can be seen in the data for strangeness multiplicities at constant beam energy and variing mass number [21]. The reason for the behaviour in Eq. (4.7) is that for larger systems the interior zone keeps together longer and thus comes closer to chemical equilibrium.

The asymptotic value of $6.5 \%$ in Eq. (4.8) is about a factor of three small than the data given in Ref. [21]. There may be several reasons for this discrepancy. The first one is geometrical: Equation 4.6 contains a factor $z^{3 / 2}$, which accounts for the smaller size of the strange system compared to the light quark system. Estimating this factor to be $z=2 / 3$ from our numerical results, this suppresses the strangeness yield by a factor two. It is probable that this factor will appear with a different power for a cylindrically symmetric system. The second reason for our low strangeness yield is that strangeness production in the initial high energy nucleon-nucleon collisions. like $p p \rightarrow p \Lambda K$, and strangeness production in the hadronic phase like $\pi \rho \rightarrow K K$ are absent from our calculation.

\section{SUMMARY AND CONCLUSIONS}

We have developed numerical methods for the solution of transport equations whose physics is derived from the two and three flavor NJL Lagrangian and have given explicit solutions for various initial conditions. A finite difference algorithm is used to solve the partial differential equation for a spherically symmetric system.

The paper is divided in two parts: First, the analytical and numerical properties of the two flavor Vlasov equation are given. Then this equation is generalized to include strangeness and also a collision term is added, so one deals with a Boltzmann type equation with selfconsistent medium dependent input.

The most serious limitation encountered is the nonrenomalizability of the NJL model, which is treated by a cutoff of $\Lambda=602 \mathrm{MeV}$. For a thermal system with $T=240 \mathrm{MeV}$ about $60 \%$ of all particles have momenta $p>\Lambda$ and cannot be properly accounted for.

For the Vlasov equation the total energy includes a potential as well as a kinetic term. The potential contributes ca. $5 \%$ to the total energy at $t=0$. During the evolution, its contribution tends to zero as the mass evolves towards its vacuum value. The expansion dynamics in the Vlasov equation is dominated by the increase 
of the constituent quark mass from $m_{q}=58 \mathrm{MeV}$ at $t=0$ to $m_{q}=312 \mathrm{MeV}$ at $t=\infty$. Correspondingly the quarks are slowed down during the expansion. For this reason the particle spectrum in momentum space changes during the expansion such that the low momentum region is enhanced and the high momentum region depleted.

Simultaneously with the introduction of the collision term, the calculation is generalized to the three flavor model in order to study also the production of strange quarks. The collision term is implemented in a relaxation time approximation. In contrast to the Vlasov equation, the Boltzmann equation leads to an energy spectrum with an exponential shape at high energies. However, the mean field still manifests itself in an enhancement of the the low energy region of the spectra like for the Vlasov equation. The shapes of the spectra do not change when the medium effects in the cross sections are switched off.

Only for large systems chemical as well as thermal equilibration of strange quarks can be reached. The approach to chemical equilibrium as a function of the initial radius $r_{0}$ can be well approximated by a $\tanh \left(a r_{0}\right)$ dependence. The absolute value of the strangeness yield found in our calculation is about a factor three smaller than observed in experiment [21].

What have we learned from this calculation? Admittedly, an expanding quark system is an artificial system, since it lacks confinement. Yet it is a system in which the dynamics of a non-equilibrium system, which underlies chiral symmetry, can be studied. Medium effects are taken care of in the mean field as well as in the collision term. In this respect the sophistication surpasses other calculations using more realistic interaction models. While we cannot compare directly with experiment, yet at least three results may be of relevance: (i) The final spectra strongly depend on the mean field. For instance it is responsible for the enhancement at small energies. This is a direct consequence of the transition from a chirally symmetric to a chirally broken phase. (ii) The calculated spectra are rather robust with respect to changes in the collision terms. Whether one uses medium dependent cross sections or Born cross sections makes little difference, provided one does not modify the masses and the mean field. (iii) Medium effects are small for the masses of the strange quarks. The rather high values of $m_{s}$ are one reason, why strangeness production in the quark plasma alone accounts for only $30 \%$ of the observed value. 


\section{ACKNOWLEDGMENTS}

We wish to thank S. P. Klevansky for illuminating discussions. This work has been supported in part by the Deutsche Forschungsgemeinschaft under contracts no. $\mathrm{Hu}$ 233/4-4, and the Federal Ministry for Education and Research under contract no. 06 HD 742 . 


\section{REFERENCES}

[1] U. Vogl and W. Weise, Prog. Part Nucl. Phys. 27, 195 (1991); S. P. Klevansky, Rev. Mod. Phys. 64, 649 (1992); T. Hatsuda and T. Kunihiro, Phys. Rep. 247, 221 (1994).

[2] E. Laermann, Nucl. Phys. A 610, 1c (1996).

[3] W. Zhang and L. Wilets, Phys. Rev. C 45, 1900 (1992).

[4] S. P. Klevansky, A. Ogura and J. Hüfner, University of Heidelberg Report No. HD-TVP-97/02 (1997) (hep-ph/9708263), to appear in Ann. Phys. (NY).

[5] P. Rehberg, S. P. Klevansky and J. Hüfner, Nucl. Phys. A 608, 356 (1996).

[6] L. P. Kadanoff and G. Baym, Quantum Statistical Mechanics (Benjamin / Cummings, Reading, MA, 1962); P. Danielewicz, Ann. Phys. (NY) 152, 239 (1984); S. R. de Groot, W. A. van Leeuwen, Ch. G. van Weert, Relativistic Kinetic Theory (North Holland, Amsterdam, 1980); W. Botermans and R. Malfliet, Phys. Rep. 198, 115 (1990); S. Mrówczyński and U. Heinz, Ann. Phys. (NY) 229, 1 (1994).

[7] W. Florkowski, J. Hüfner, S. P. Klevansky and L. Neise, Ann. Phys. (NY) 245, 445 (1995).

[8] E. Quack and S. P. Klevansky, Phys. Rev C 49, 3283 (1994); J. Müller and S. P. Klevansky, Phys. Rev. C 50, 410 (1994); V. Dmitrašinović, H.-J. Schulze, R. Tegen and R.H. Lemmer, Ann. Phys. (NY) 238, 332 (1995).

[9] J. Hüfner, S. P. Klevansky, P. Zhuang and H. Voss, Ann. Phys. (NY) 234, 225 (1993); P. Zhuang, J. Hüfner and S. P. Klevansky, Nucl. Phys. A 576, 525 (1994).

[10] W. Florkowski, Phys. Rev. C 50, 3069 (1994).

[11] R. W. Hockney and J. W. Eastwood, Computer Simulation Using Particles (IOP Publishing Ltd., Bristol, 1988).

[12] A. Abada and J. Aichelin, Phys. Rev. Lett. 74, 3130 (1995). 
[13] W. H. Press, B. P. Flannery, S. A. Teukolsky and W.T. Vetterling, Numerical Recipes, 2nd ed. (Cambridge University Press, 1992).

[14] P. Zhuang, J. Hüfner, S. P. Klevansky and L. Neise, Phys. Rev. D 51, 3728 (1995).

[15] P. Rehberg, S. P. Klevansky and J. Hüfner, Phys. Rev. C 53, 410 (1996).

[16] J. D. Bjorken and S. D. Drell, Relativistic Quantum Fields (McGraw Hill, New York, 1965); C. Itzykson and J.-B. Zuber, Quantum Field Theory, (McGraw Hill, New York, 1980).

[17] J. Hüfner, S. P. Klevansky and P. Rehberg, Nucl. Phys. A 606, 260 (1996).

[18] N. Herrmann, Nucl. Phys. A 610, 49c (1996).

[19] I. G. Bearden et. al., Phys. Rev. Lett. 78, 2080 (1997).

[20] T. Csörgő and B. Lörstad, Phys. Rev. C 54, 1390 (1996).

[21] M. Gaździcky and D. Röhrich, Z. Phys. C 71, 55 (1996). 


\section{TABLES}

TABLE I. Particle multiplicities for different settings of initial system radius and maximal momentum. In row (c), high momenta are cut off by a multiplicative factor.

\begin{tabular}{c||c||c|c|c||c|c}
\hline \hline & $p_{\max } / \Lambda$ & $\mathcal{N}_{q, t=0}$ & $\mathcal{N}_{q, t=\infty}$ & $\mathcal{N}_{s, t=\infty}$ & $\mathcal{N}_{s, t=\infty} / \mathcal{N}_{q, t=\infty}$ & $\left(\mathcal{N}_{q}+\mathcal{N}_{s}\right)_{t=\infty} / \mathcal{N}_{q, t=0}$ \\
\hline (a) & 1 & 343.2 & 365.6 & 8.1 & $2.2 \%$ & 1.089 \\
(b) & 3 & 811.2 & 825.4 & 32.4 & $3.9 \%$ & 1.058 \\
(c) & $1^{*}$ & 251.2 & 242.3 & 5.7 & $2.3 \%$ & 0.987 \\
\hline \hline
\end{tabular}

TABLE II. Dependence of strangeness yield and slope temperatures on the medium effects.

\begin{tabular}{c||c|c||c|c|c}
\hline \hline & Mean Field & Cross Sections & $\mathcal{N}_{s, t=\infty} / \mathcal{N}_{q, t=\infty}$ & $T_{q}(\mathrm{MeV})$ & $T_{s}(\mathrm{MeV})$ \\
\hline (a) & yes & exact & $2.2 \%$ & 185 & 153 \\
(d) & yes & Born & $3.0 \%$ & 179 & 153 \\
(e) & no & Born & $5.5 \%$ & 207 & 191 \\
\hline \hline
\end{tabular}




\section{FIGURES}

FIG. 1. The effective Potential $\mathcal{V}_{\text {eff }}$ as a function of the constituent quark mass $m_{q}$. The parameters used for this plot are $m_{0 q}=5 \mathrm{MeV}, \Lambda=653.3 \mathrm{MeV}$ and $G \Lambda^{2}=2.10$. The constituent quark mass in the vacuum for this parameter set is $312 \mathrm{MeV}$.

FIG. 2. The radial dependence of the quarks daensity as a function of $r$ at various times $t$ during the expansion process.

FIG. 3. The radial dependence of the quark masses at various times $t$ during the expansion process.

FIG. 4. Momentum spectra for quarks in the beginning ( $t=0$, dashed line) and at the end $(t=\infty$, solid line) of the expansion.

FIG. 5. Time dependence of kinetic energy (dashed line) and total energy (solid line). Note the broken scale on the energy axis.

FIG. 6. Inverse relaxation times $\tau_{q q}^{-1}$ (solid line), $\tau_{s s}^{-1}$ (dashed line) and $\tau_{q s}^{-1}$ (dotted line) as a function of temperature for $p=200 \mathrm{MeV}$. The dot-dashed line shows $\tau_{q q}^{-1}$ in Born approximation.

FIG. 7. Inverse relaxation time $\tau_{q q}^{-1}$ as a function of temperature for $p=0$ (solid line), $p=200 \mathrm{MeV}$ (dashed line) and at $p=0$ in Born approximation (dotted line).

FIG. 8. Light quark density at the same times as in Fig. 2 for the full calculation containing three flavors and collisions.

FIG. 9. Strange quark density for the same times as in Fig. 2 for the full calculation containing three flavors and collisions. Note that the density is zero at $t=0$ and rises until $t=4 \mathrm{fm} / c$ due to production by the process $q \bar{q} \rightarrow s \bar{s}$.

FIG. 10. Light and strange quark masses as a function of time. 
FIG. 11. Distributions of light and strange quarks for the final state as a function of the particle energy (solid lines). The dashed lines are exponential fits with the slope temperatures $T_{q}=185 \mathrm{MeV}$ for light quarks and $T_{s}=164 \mathrm{MeV}$ for strange quarks.

FIG. 12. Distributions of light quarks resulting from different treatments of the high momentum part. Solid line: only particles with momentum $p \leq \Lambda$ are included. Dashed line: particles with momentum $p \leq 3 \Lambda$ are included. Dotted line: smooth cutoff of high momentum particles.

FIG. 13. Distributions of strange quarks resulting from different treatments of the high momentum part. Solid line: only particles with momentum $p \leq \Lambda$ are included. Dashed line: particles with momentum $p \leq 3 \Lambda$ are included. Dotted line: smooth cutoff of high momentum particles.

FIG. 14. Light quark spectra for three different treatments of medium effects; (a): standard procedure (solid line), (d): cross sections in Born approximation (dashed line) and (e): cross sections in Born approximation and mean field switched off.

FIG. 15. Slope temperatures for light quarks (diamonds) and strange quarks (crosses) as a function of the radius $r_{0}$ initial fireball.

FIG. 16. Variation of the strangeness yield as a function of the initial radius (diamonds). The solid line is a fit by a hyperbolic tangent. 


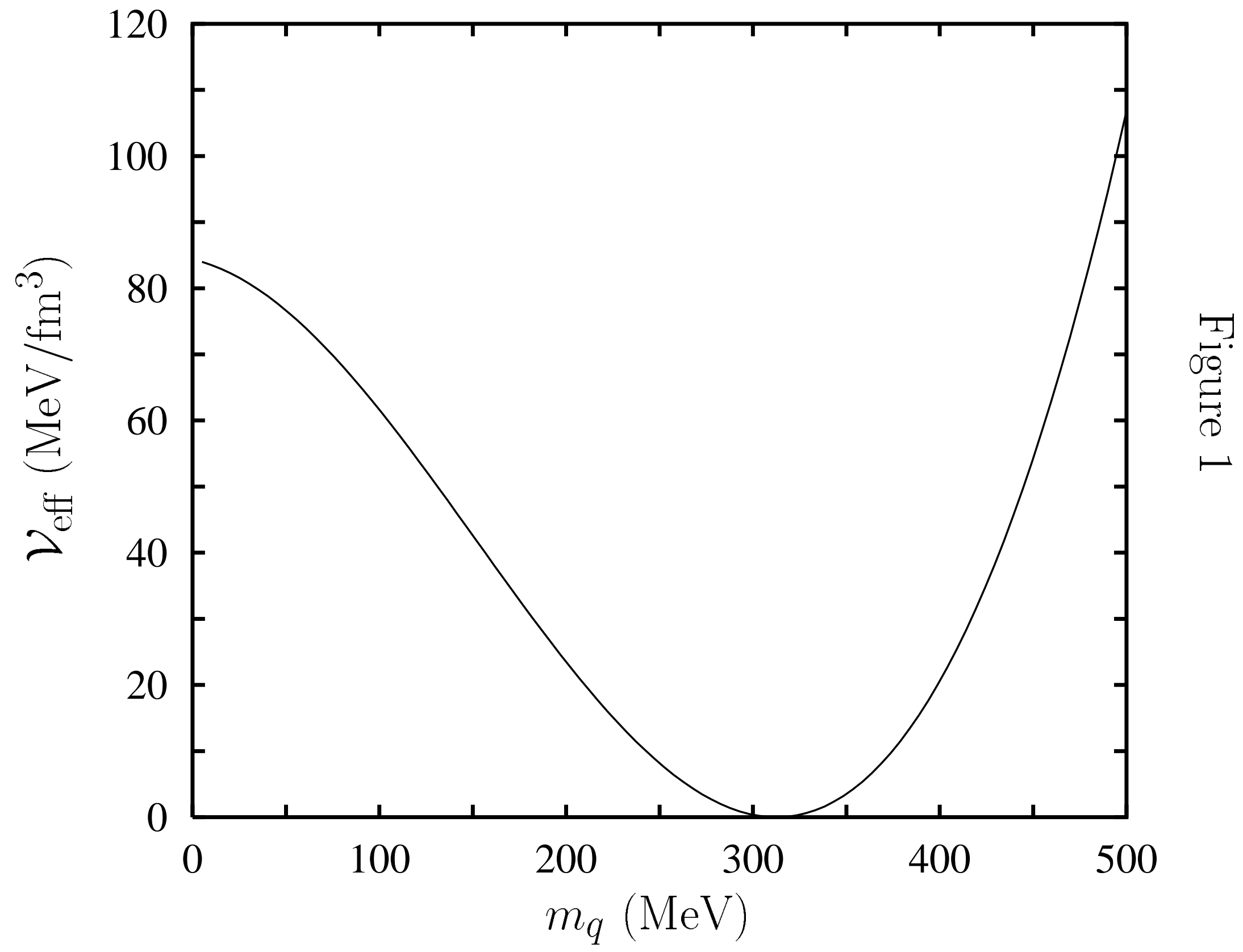




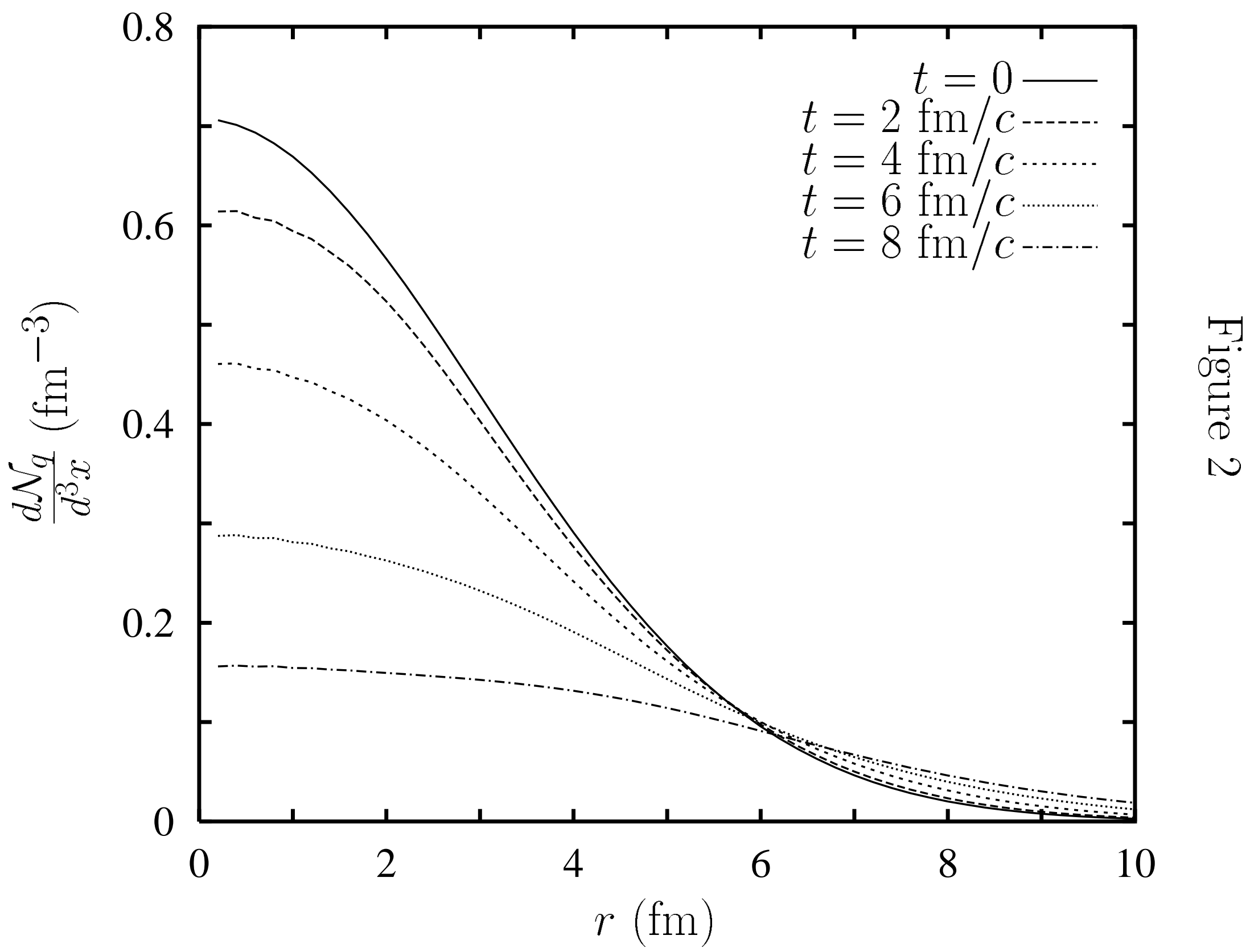




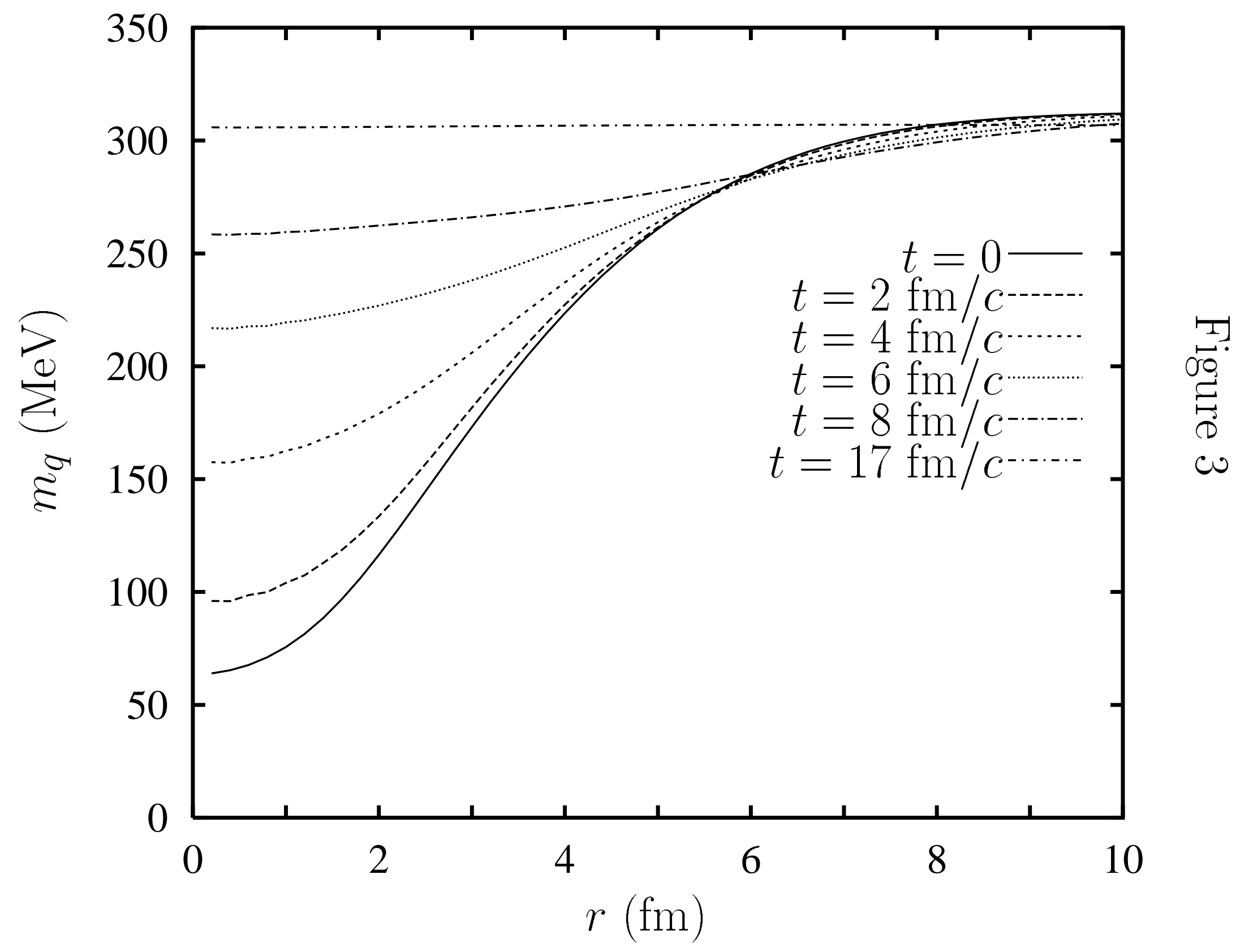




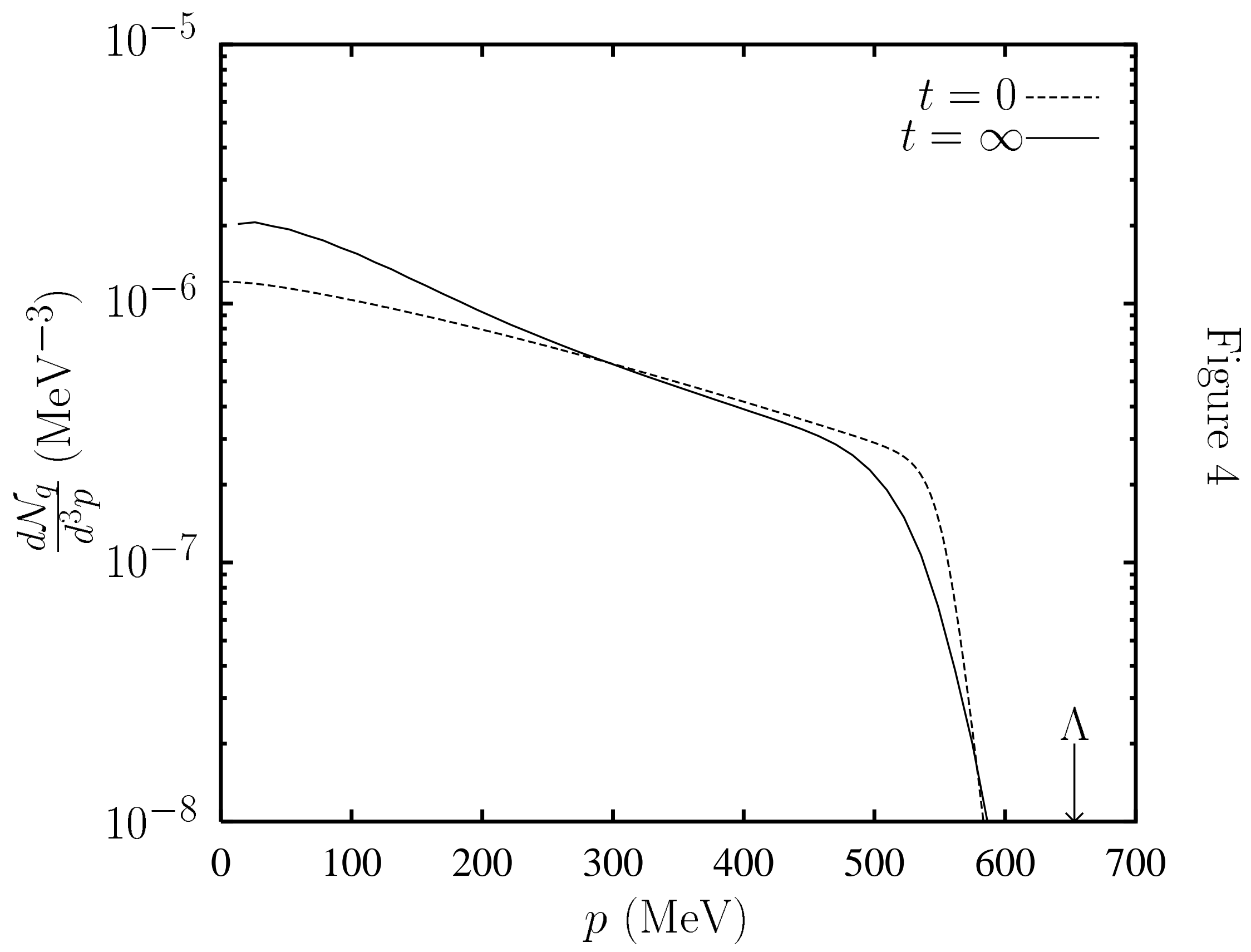




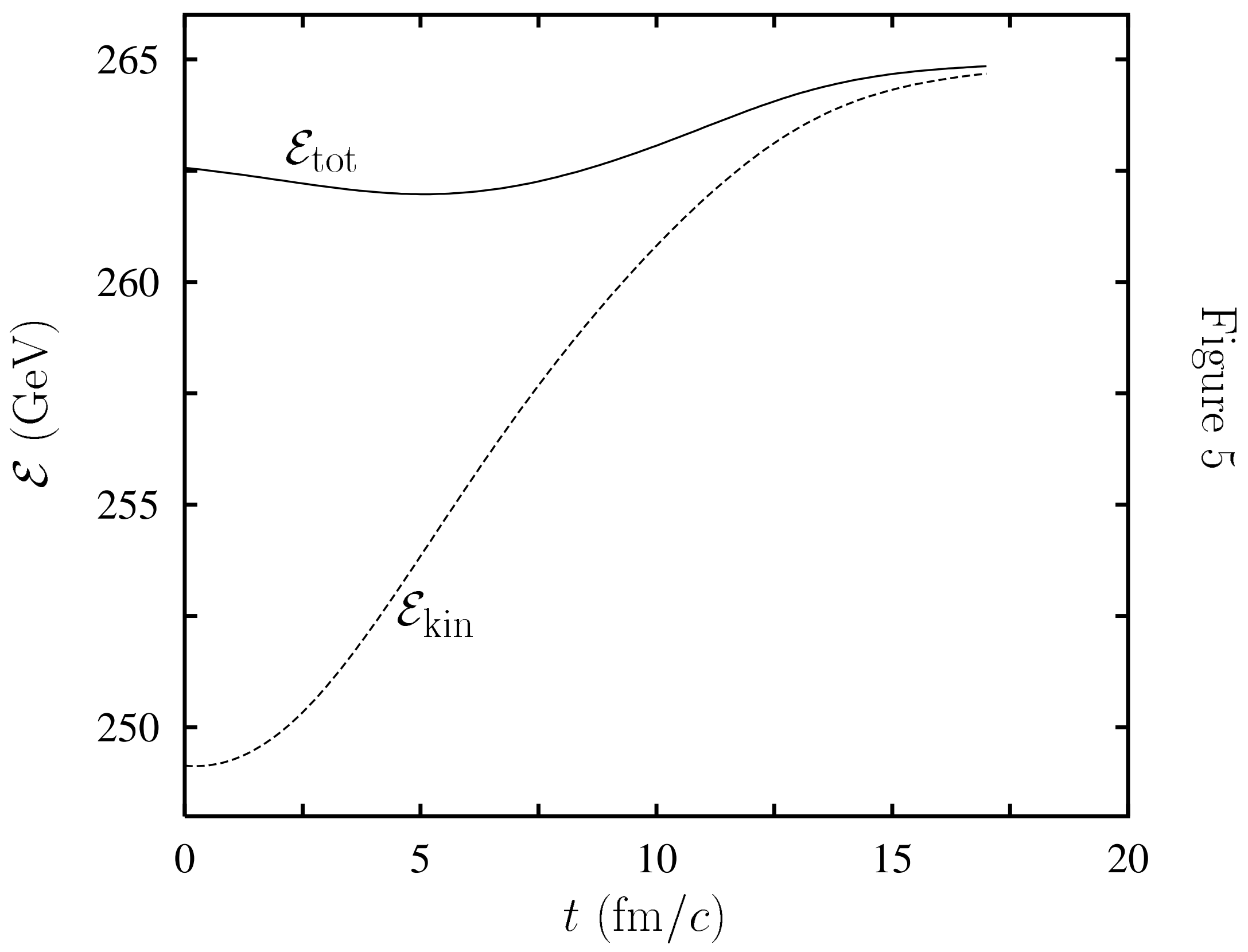


Figure 6

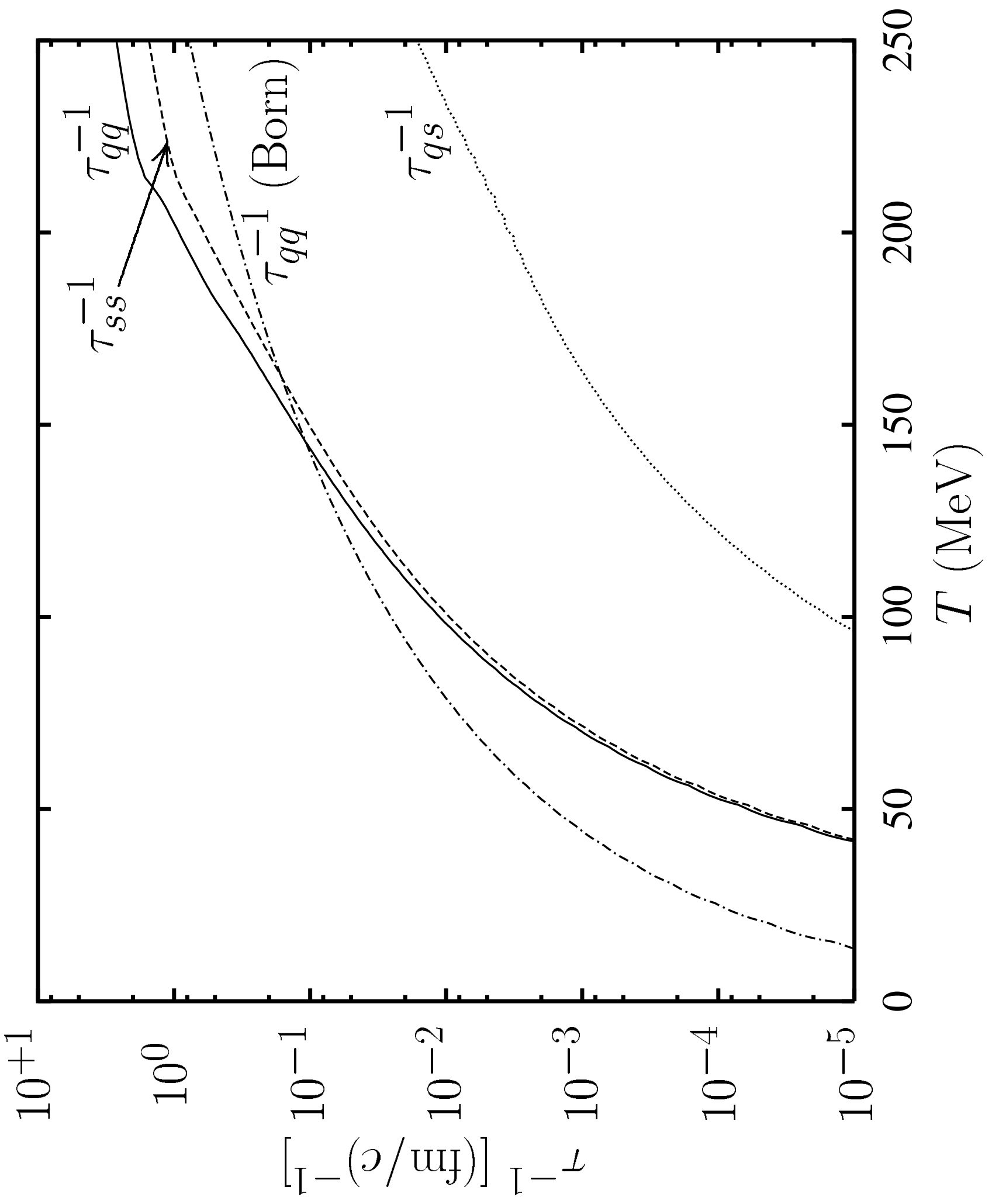




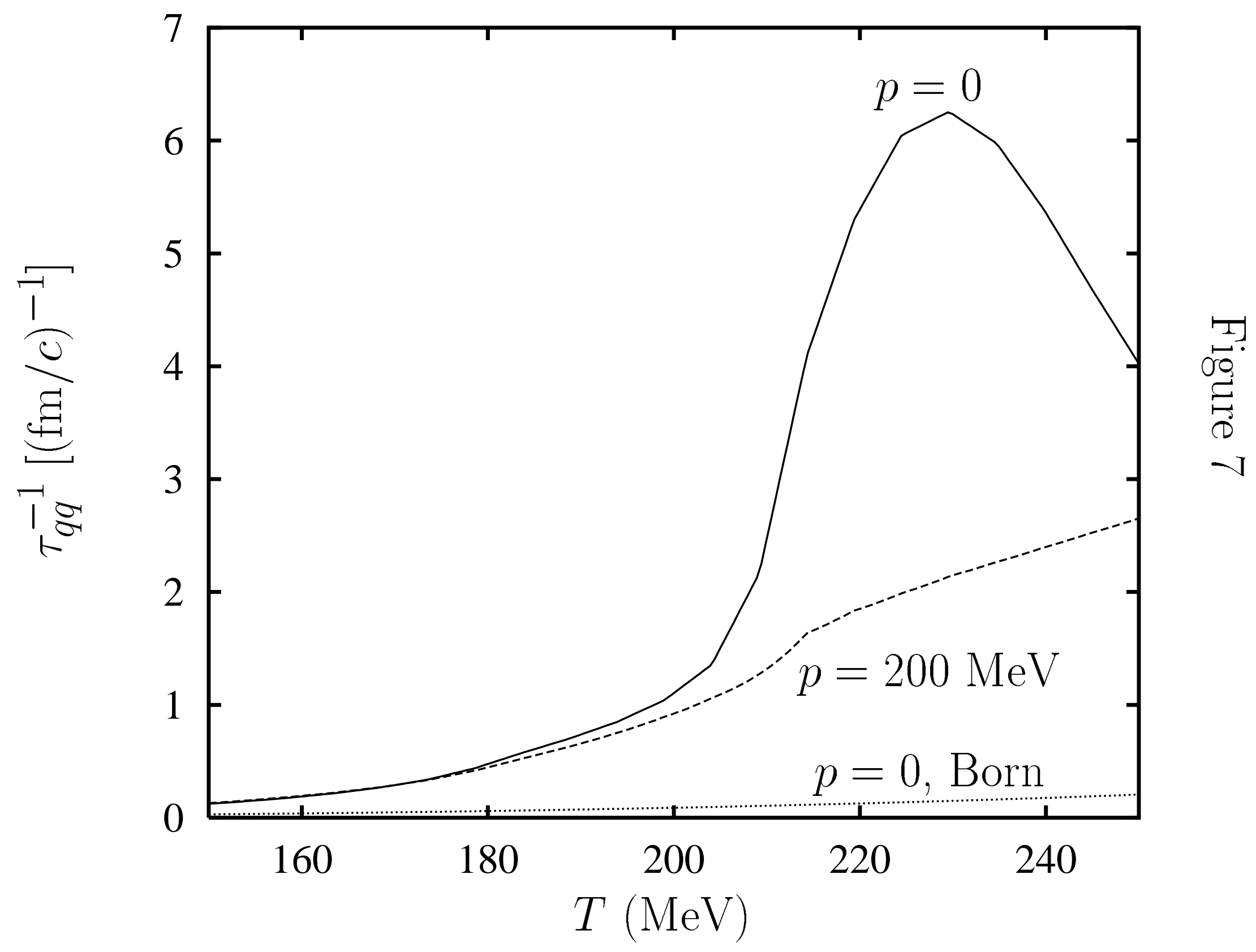




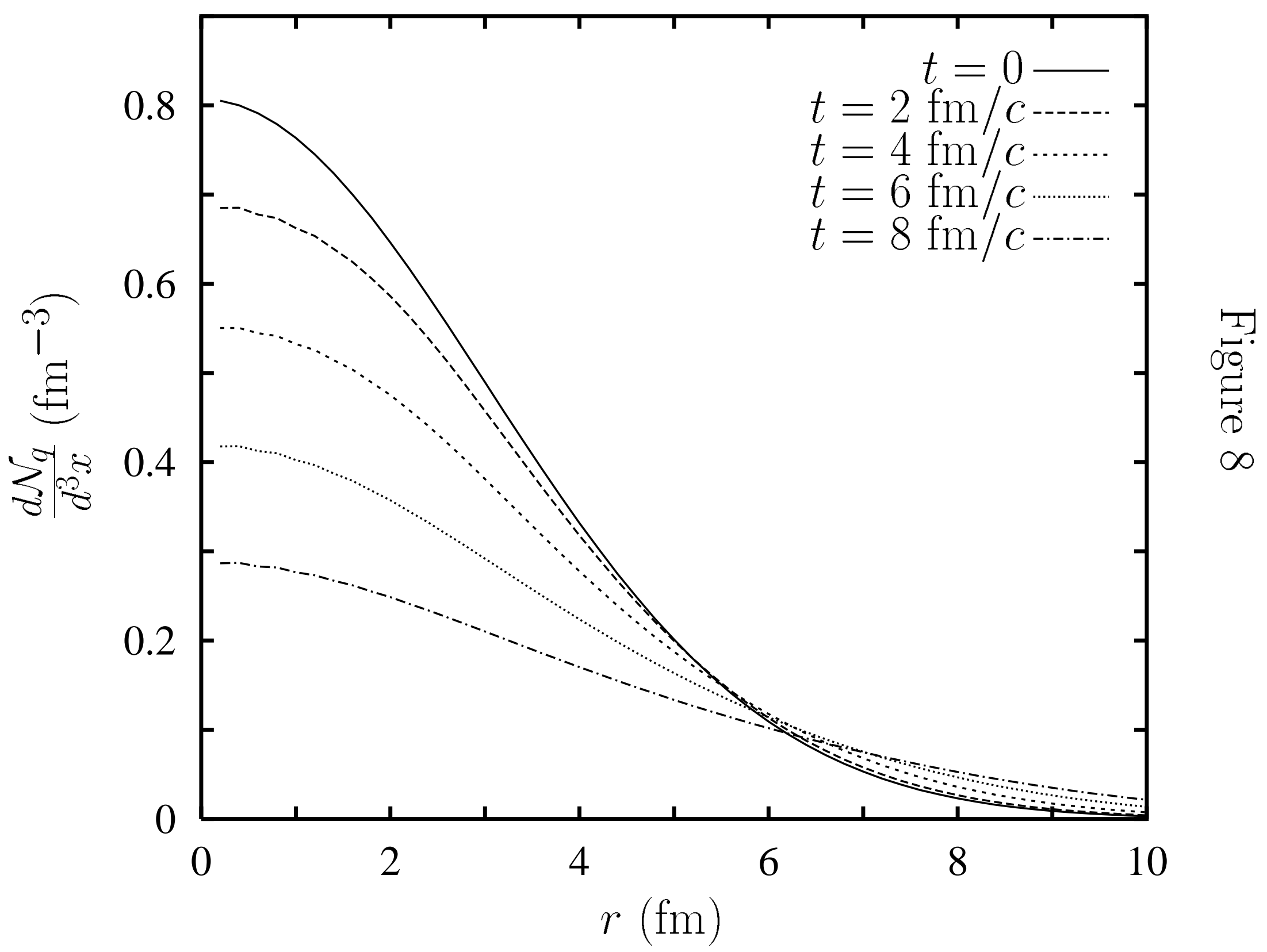




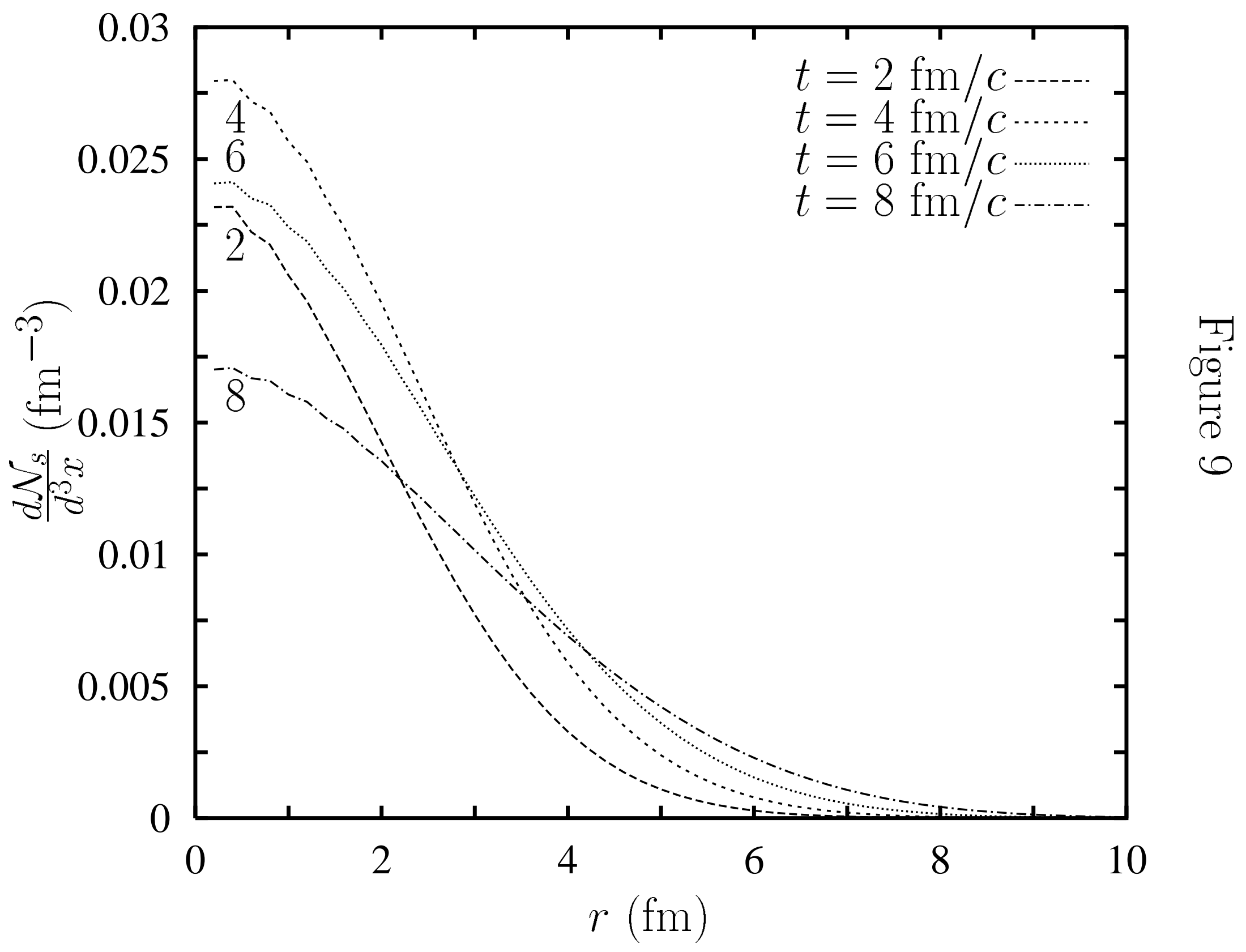




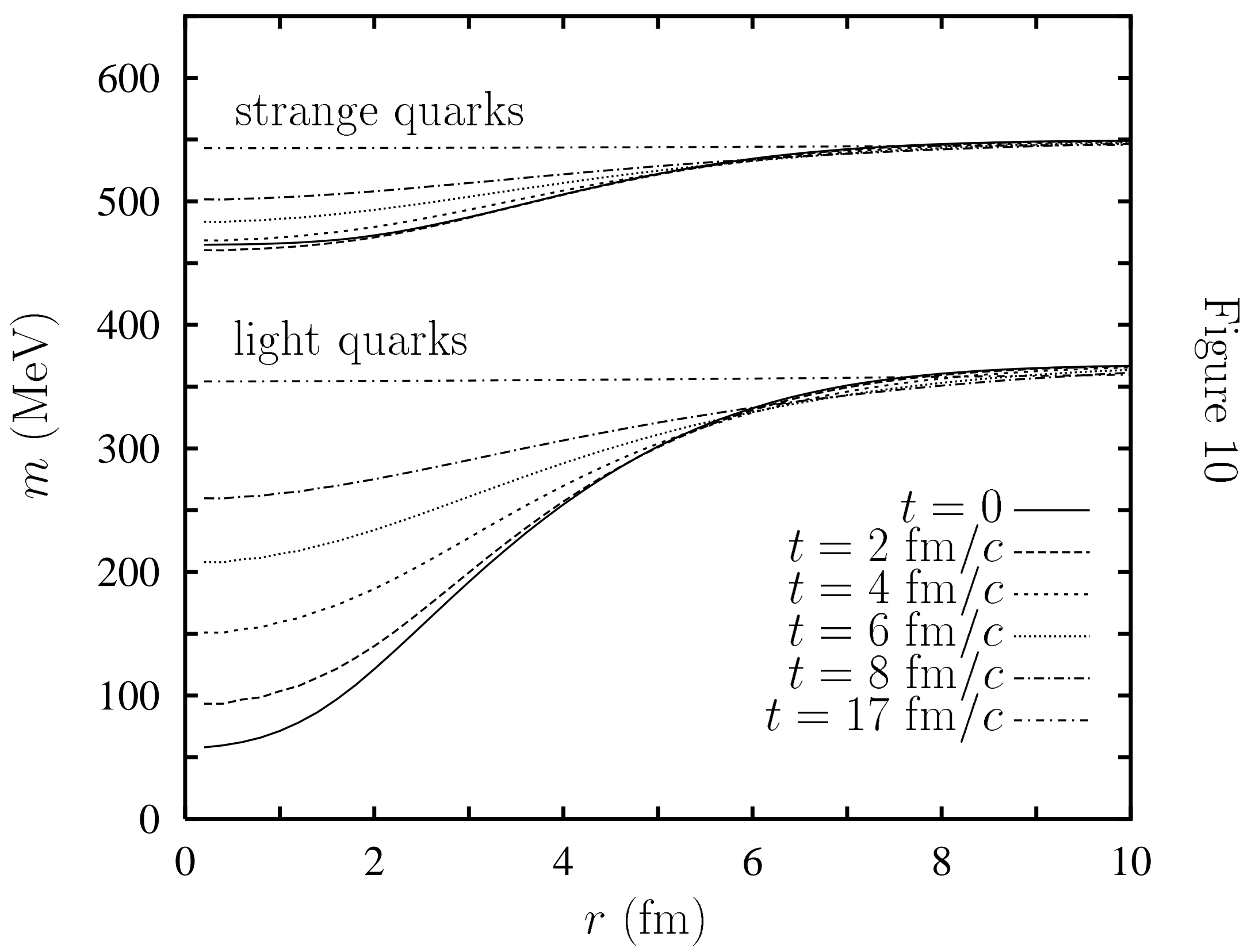




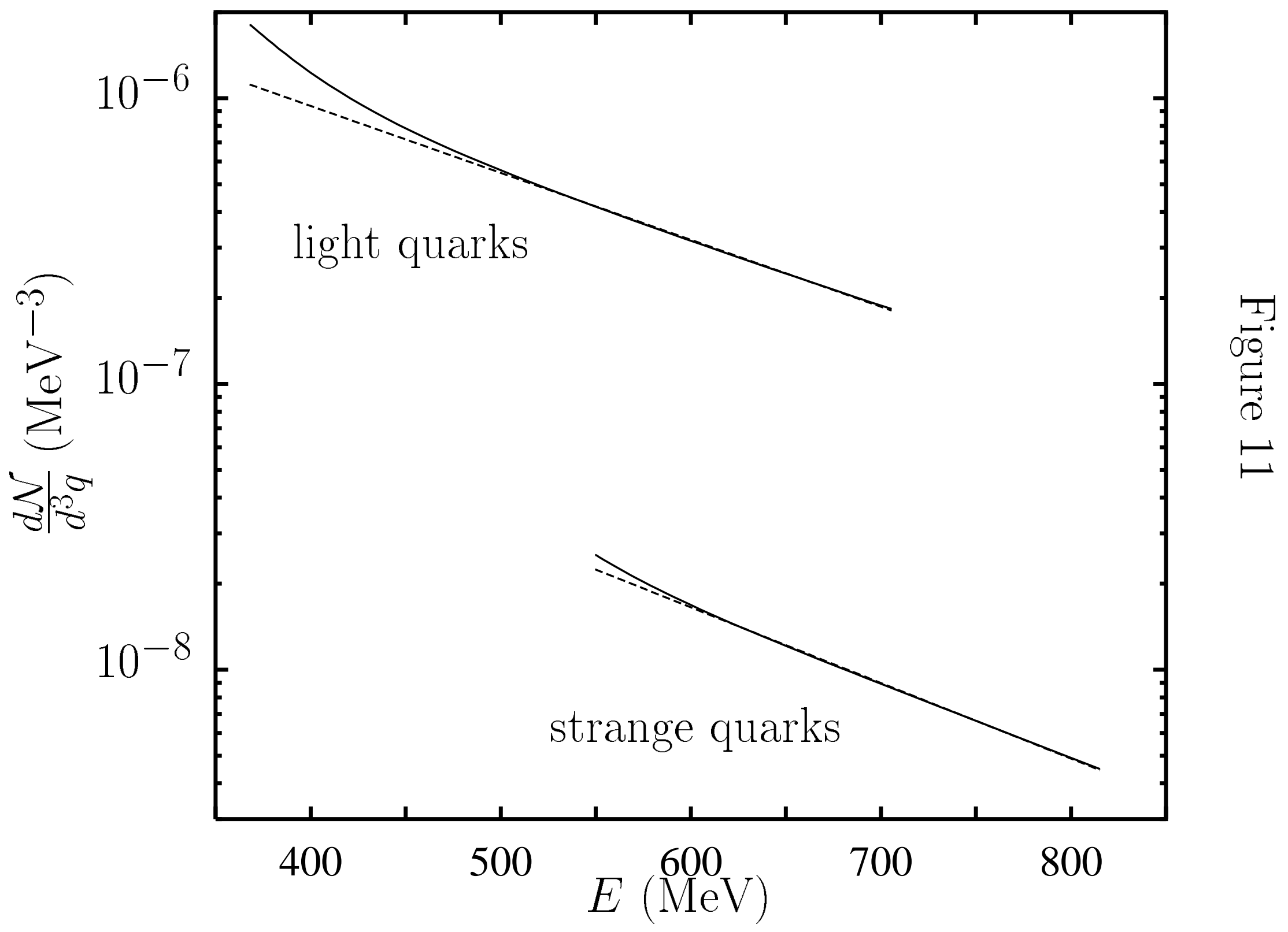




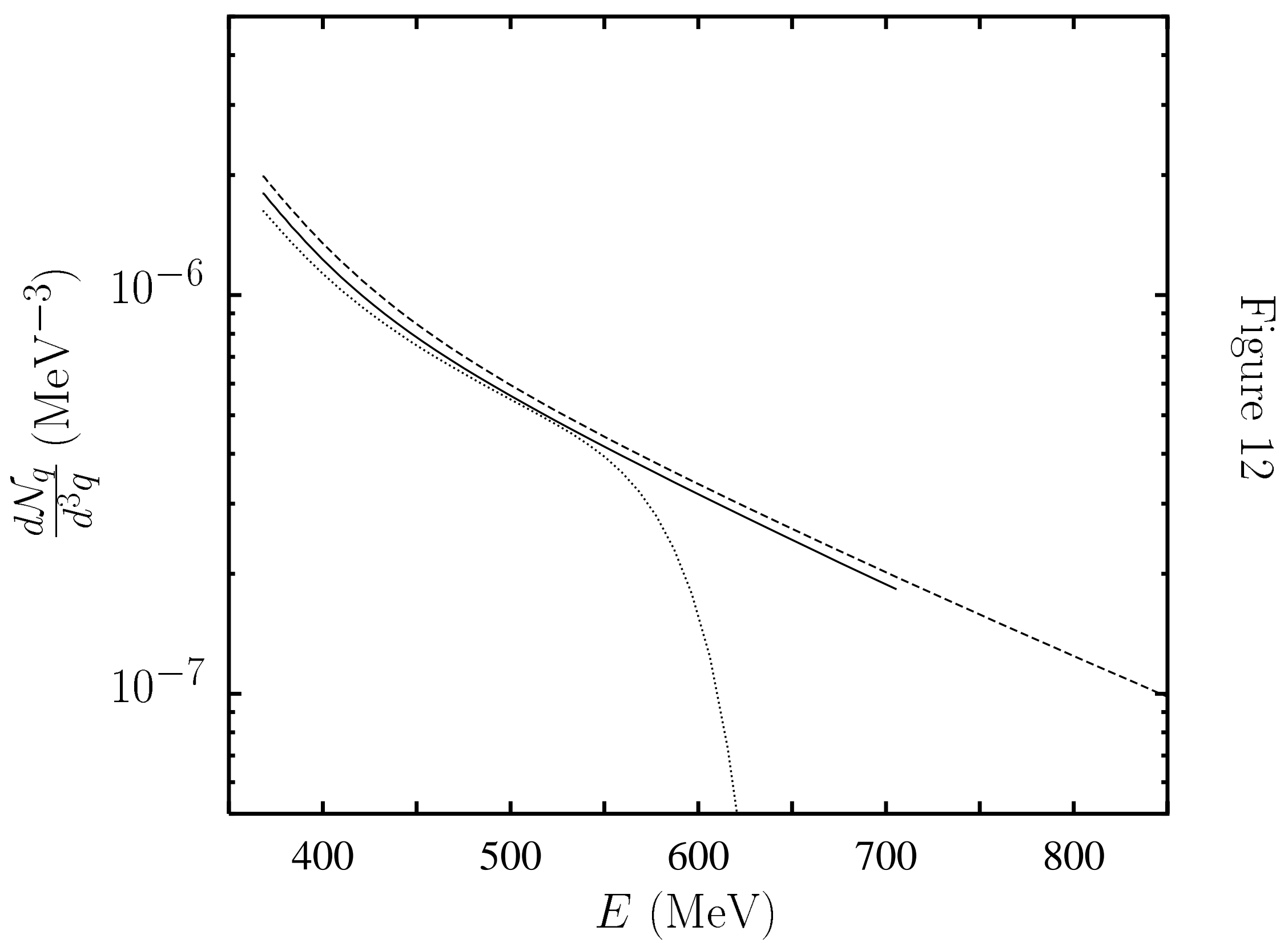




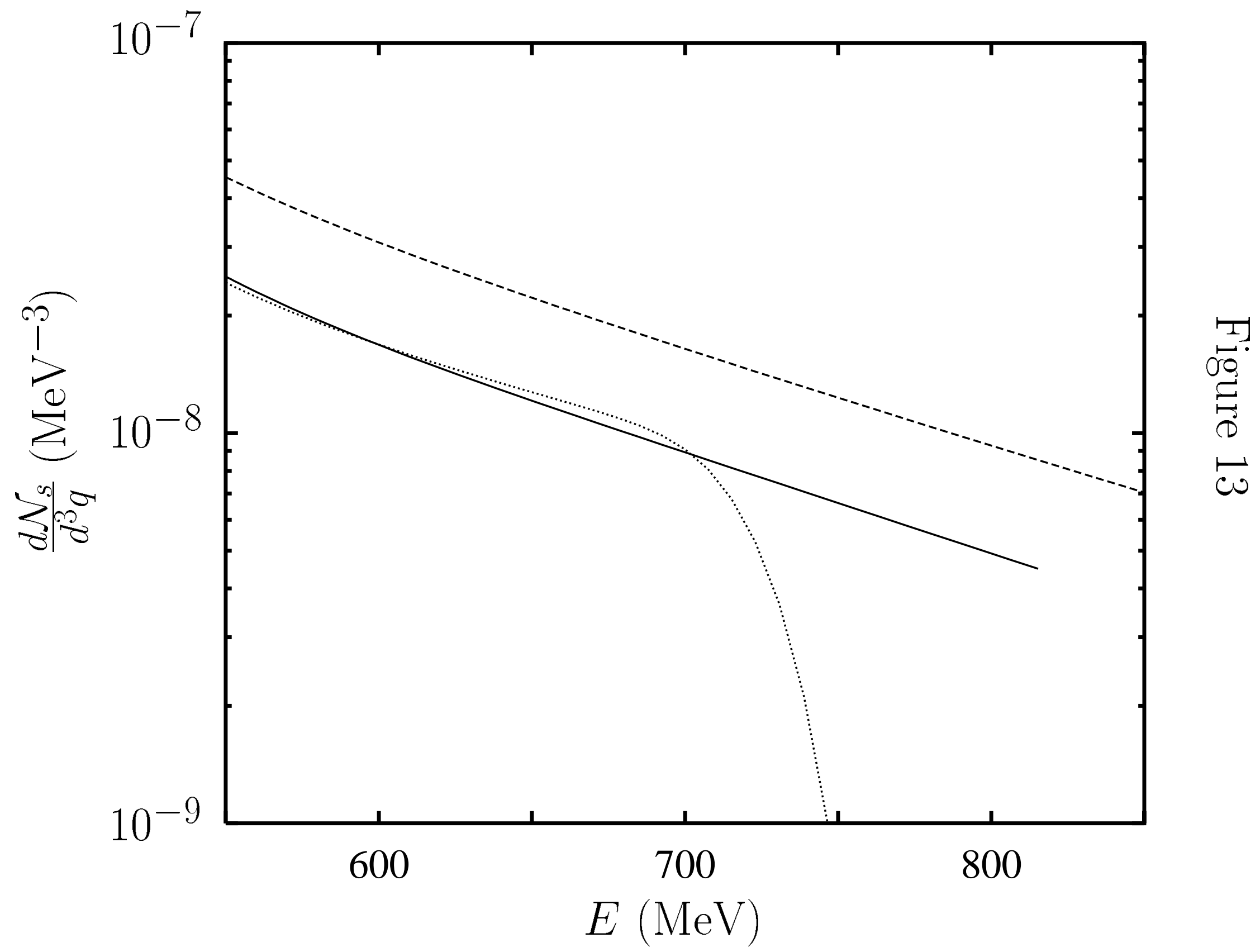




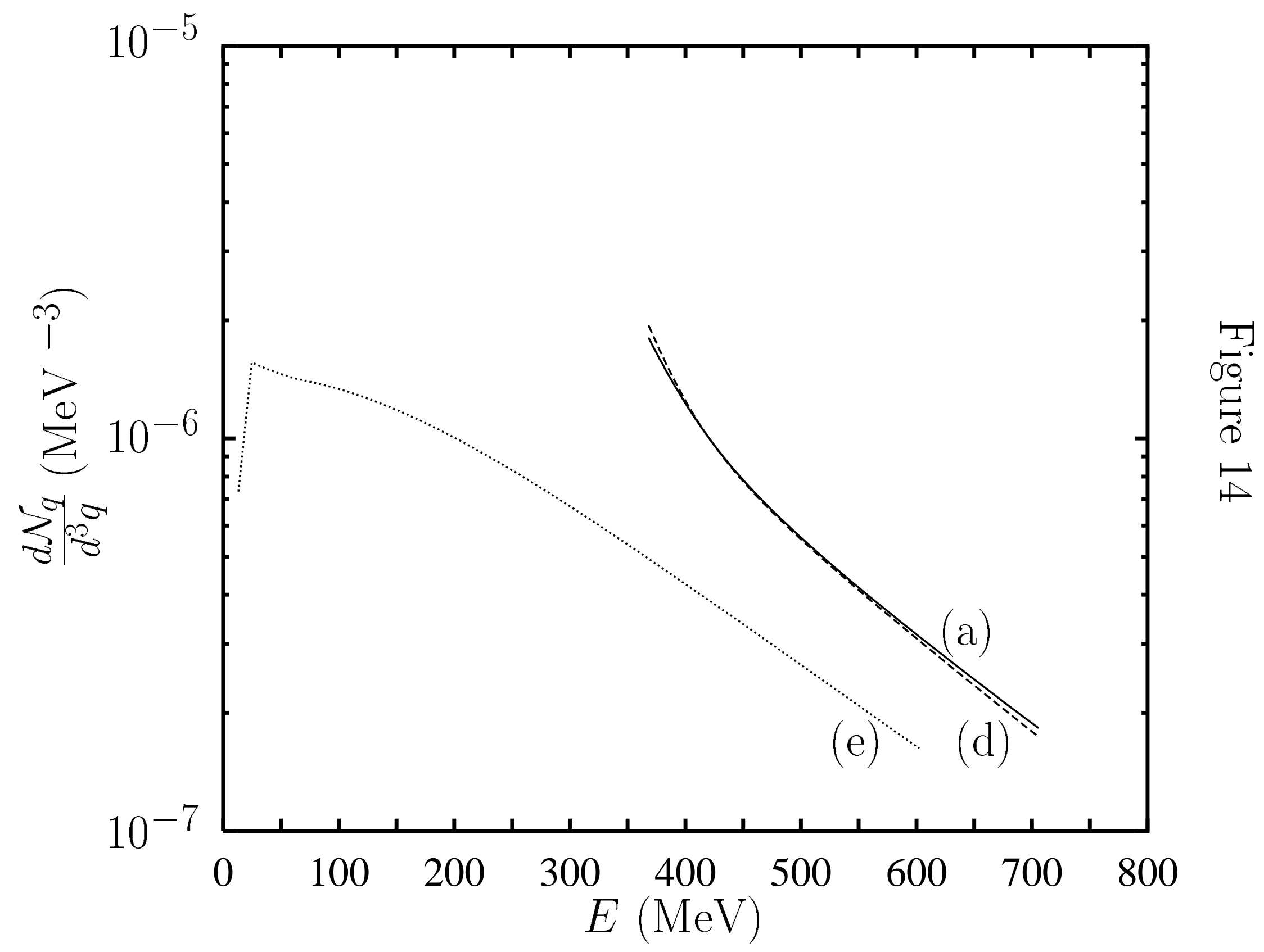




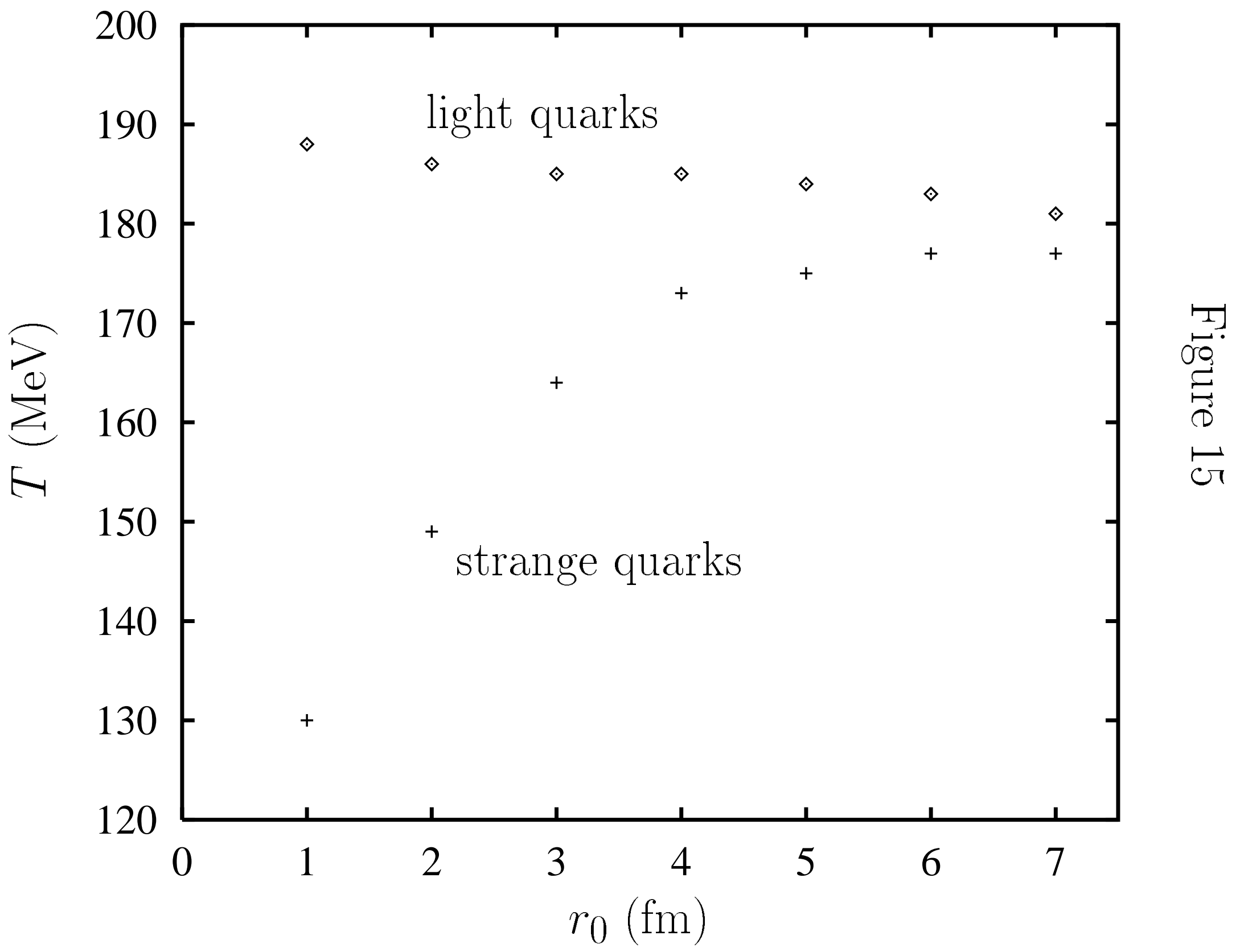




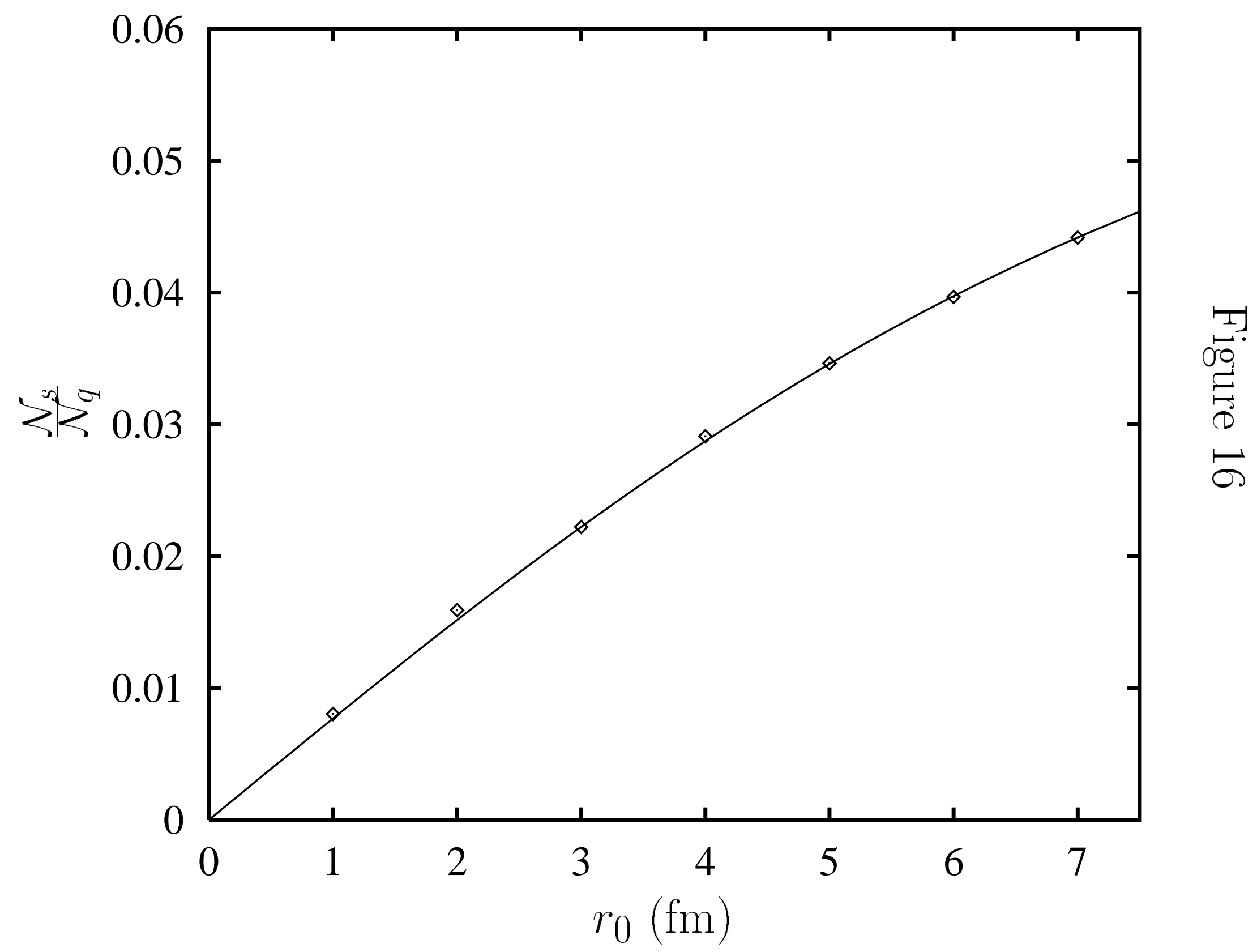

